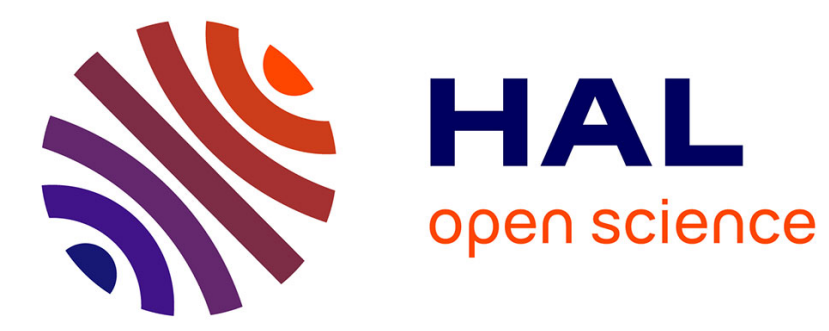

\title{
Acoustic scattering by elastic cylinders of elliptical cross-section and splitting up of resonances
}

Stéphane Ancey, Emmanuelle Bazzali, Paul Gabrielli, Michaël Mercier

\section{To cite this version:}

Stéphane Ancey, Emmanuelle Bazzali, Paul Gabrielli, Michaël Mercier. Acoustic scattering by elastic cylinders of elliptical cross-section and splitting up of resonances. Journal of Applied Physics, 2014, pp.194904. 10.1063/1.4876678 . hal-01150381

\section{HAL Id: hal-01150381 \\ https://hal.science/hal-01150381}

Submitted on 11 May 2015

HAL is a multi-disciplinary open access archive for the deposit and dissemination of scientific research documents, whether they are published or not. The documents may come from teaching and research institutions in France or abroad, or from public or private research centers.
L'archive ouverte pluridisciplinaire HAL, est destinée au dépôt et à la diffusion de documents scientifiques de niveau recherche, publiés ou non, émanant des établissements d'enseignement et de recherche français ou étrangers, des laboratoires publics ou privés. 


\section{Acoustic scattering by elastic cylinders of elliptical cross-section and splitting up of resonances}

S. Ancey, ${ }^{1, a)}$ E.Bazzali, ${ }^{1, b)}$ P. Gabrielli, ${ }^{1,}$ c) and M.Mercier ${ }^{1, d}$ )

UMR CNRS 6134 SPE, Faculté des Sciences, Université de Corse, F-20250 Corte, France

(Dated: 12 March 2014)

The scattering of a plane acoustic wave by an infinite elastic cylinder of elliptical cross section is studied from a modal formalism by emphasizing the role of the symmetries. More precisely, as the symmetry is broken in the transition from the infinite circular cylinder to the elliptical one, the splitting up of resonances is observed both theoretically and experimentally. This phenomenon can be interpreted using group theory. The main difficulty stands in the application of this theory within the framework of the vectorial formalism in elastodynamics. This method significantly simplifies the numerical treatment of the problem, provides a full classification of the resonances and gives a physical interpretation of the splitting up in terms of symmetry breaking.

An experimental part based on ultrasonic spectroscopy complements the theoretical study. A series of tank experiments is carried out in the case of aluminium elliptical cylinders immersed in water, in the frequency range $0 \leq k r \leq 50$, where $k r$ is the reduced wave number in the fluid. The symmetry is broken by selecting various cylinders of increasing eccentricity. More precisely, the greater the eccentricity, the higher the splitting up of resonances is accentuated. The experimental results provide a very good agreement with the theoretical ones, the splitting up is observed on experimental form functions and the split resonant modes are identified on angular diagrams.

PACS numbers: 43.20.Fn, 43.20.Ks, 46.40.Cd, 02.20.-a

Keywords: acoustic scattering, resonances, splitting, elastodynamics, symmetry breaking, ultrasonic spectroscopy

\footnotetext{
a) Electronic mail: ancey@univ-corse.fr.

b) Electronic mail: ebazzali@univ-corse.fr.

c)Electronic mail: gabrieli@univ-corse.fr.

d)Electronic mail: mercier@univ-corse.fr.
} 


\section{INTRODUCTION}

The scattering by objects of simple shapes has been extensively studied during the last 50 years. Most of the work performed on scattering by cylindrical objects has focused on the restricted case of cylinders having a circular cross section ${ }^{1}$. In comparison, little attention has been given to the more general case of the elliptical cylindrical geometries ${ }^{2}$, especially in the experimental field. Experimental results for scatterers of non circular cross section can be found $\mathrm{in}^{3,4}$. More recently, Léon et al. ${ }^{5,6}$ used a modal formalism for investigating the acoustic scattering by elastic cylinders of arbitrary cross section immersed in a fluid and compared their theoretical and experimental results.

Various methods can be applied to treat the scattering problem in the case of non circular geometry. The theoretical studies based on the expansion in Mathieu functions ${ }^{7-9}$ are well suited for elliptical cylinders but in elastodynamics, one has to deal with alternative methods because Helmholtz equation does not separate in elliptical coordinates, due to the existence of longitudinal and transverse waves ${ }^{10,11}$. The T-matrix method is efficient to deal with elastic scatterers in any noncircular cylindrical geometries ${ }^{11-15}$. The modal formalism applied in ${ }^{5}$ by Léon et al. is close to this approach. We can also cite the boundary integral methods based on the Green's function approach, leading to the so-called Fredholm integral equations $^{16,17}$ and a method involving conformal mappings, the Fourier Matching Method, described by DiPerna and Stanton ${ }^{18}$.

The present work deals with the scattering of a plane acoustic wave by infinite elastic cylinder of elliptical cross section from a modal formalism associated with symmetry considerations. The problem is studied theoretically, numerically and experimentally. Interest is focused on the splitting up of resonances which occurs when the symmetry is broken in the transition from the circular cylinder to the elliptical one. In terms of group theory, this corresponds to the symmetry breaking $\mathcal{O}(2) \rightarrow \mathcal{C}_{2 v}$. This splitting up has been numerically observed for the first time by Moser and Überall ${ }^{19}$. More recently, Chinnery and Humphrey discussed about mode splittings and level crossings in the study of the acoustic resonances of water-filled cylindrical shells of elliptical cross section ${ }^{3}$. However, none of these authors provides an explanation or analytical description to this phenomenon. Ancey et $a l^{20-22}$ have highlighted and explained the splitting up of resonances in the elliptical geometry using a method involving group theory ${ }^{23}$. This technique has been also used to study multiple 
scattering ${ }^{24,25}$. The main advantages of this method stand in (i) the uncoupling of the equations, (ii) the classification of resonances, (iii) the highlighting of the splitting up of resonances and its interpretation in terms of symmetry breaking and (iv) the improvement in numerical computations.

The paper is organized as follows. In section II, the geometry of the problem is presented and algebraic considerations are recalled, particularly the symmetry transformations. In section III, a modal formalism in polar coordinates combined with group theory is applied to express the boundary conditions. They lead to four infinite systems of equations, each one associated with a given irreducible representation of the symmetry group $\mathcal{C}_{2 v}$. The scattering problem is uncoupled and these systems can be solved numerically by truncation and used to obtain the resonances, the elastic displacement, the scattered pressure and the far field form function. Numerical and experimental results are investigated in section IV. The numerical evaluations of resonances and backscattered total form functions are carried out in the cases of aluminium elliptical cylinders immersed in water. A series of experiments based on ultrasonic spectroscopy is performed and the results are compared with the theoretical ones in a quite good agreement. The splitting up of resonances is observed on both theoretical and experimental form functions. The amplitudes of the elastic displacement and the scattered pressure are computed and plotted, they also highlight the mode splittings. Finally, angular diagrams confirm experimentally the identification of the resonant mode studied. In section $\mathrm{V}$, the interest of the method used in this paper is highlighted and future extensions in scattering and elastodynamics are suggested.

\section{POSITION AND GEOMETRY OF THE PROBLEM}

\section{A. Position of the problem}

The scattering of plane acoustic wave by an infinite elastic cylinder of elliptical cross section is studied. The cylinder is immersed in a homogeneous fluid of density $\rho_{f}$ and sound velocity $c$. The elastic medium is characterized by the density $\rho_{s}$ and the longitudinal and transverse velocities $c_{L}$ and $c_{T}$. We introduce the wave numbers $k_{L}=\omega / c_{L}$ and $k_{T}=\omega / c_{T}$, where $\omega$ is the angular frequency. The time dependence $e^{-i \omega t}$ is assumed throughout the paper. The $z$-axis is taken parallel to the axis of the cylinder and $(\rho, \theta)$ are the polar 
coordinates in a plane perpendicular to $z$. The propagation vector $\mathbf{k}$ of the incident plane wave is perpendicular to the $z$-axis and forms an angle $\alpha$ with the $x$-axis. Then we shall assume that the problem is independent of the $z$-coordinate and thus reduces to a twodimensional one. The geometry as well as the notations used are displayed in Fig. 1. The boundary is a closed elliptical curve described by the radius $r(\theta)$ with a continuously turning outward normal $\mathbf{n}(\theta)$ defined by

$$
\mathbf{n}(\theta)=n_{\rho} \mathbf{e}_{\rho}+n_{\theta} \mathbf{e}_{\theta}
$$

with

$$
n_{\rho}=\frac{1-e^{2} \cos ^{2} \theta}{\sqrt{1+e^{2}\left(e^{2}-2\right) \cos ^{2} \theta}}, \quad n_{\theta}=\frac{e^{2}}{2} \frac{\sin 2 \theta}{\sqrt{1+e^{2}\left(e^{2}-2\right) \cos ^{2} \theta}}
$$

and

$$
r(\theta)=\frac{b}{\sqrt{1-e^{2} \cos ^{2} \theta}}
$$

where $e^{2}=1-\frac{b^{2}}{a^{2}}$ defines the eccentricity $e$ (Fig. 1).

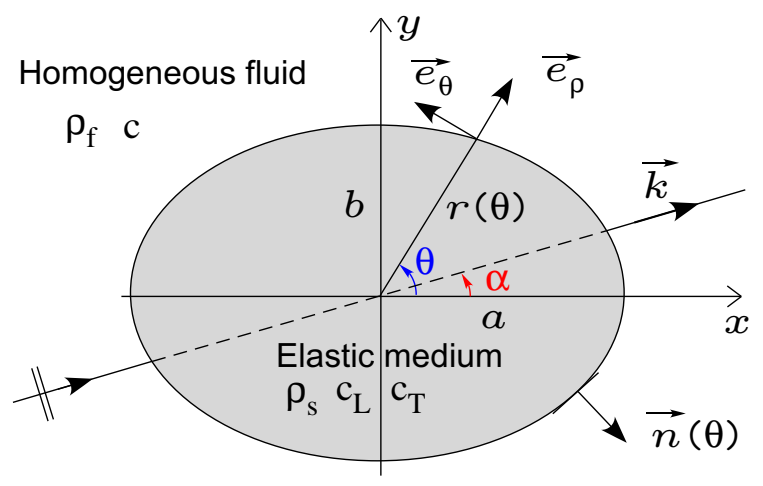

FIG. 1. Geometry of the problem.

The incident wave is expressed in terms of acoustic pressure as

$$
p_{\text {inc }}=\sum_{n=-\infty}^{+\infty} i^{n} J_{n}(k \rho) e^{i n(\theta-\alpha)}
$$

The scattered pressure must statisfy the Sommerfeld's radiation condition at infinity. Then, it can be expressed as

$$
p_{s}=\sum_{n=-\infty}^{+\infty} i^{n} A_{n}^{S} H_{n}^{(1)}(k \rho) e^{i n \theta}
$$

where $A_{n}^{S}$ are unknown coefficients to be determined. 
The elastic displacement $\mathbf{u}$ is expressed using the Helmholtz decomposition:

$$
\mathbf{u}=-\nabla \phi+\nabla \wedge \psi
$$

where $\phi$ and $\boldsymbol{\psi}=\psi \mathbf{e}_{z}$ are the scalar and vectorial potentials respectively associated with the longitudinal and transverse fields. The scalar potentials $\phi$ and $\psi$ satisfy the Helmholtz equations $\nabla^{2} \phi+k_{L}^{2} \phi=0$ and $\nabla^{2} \psi+k_{T}^{2} \psi=0$. Since the longitudinal and transverse fields in the elastic elliptical cylinder have no singularity in the neighbourhood of the origin, they can be expanded in terms of Bessel functions as

$$
\phi=\frac{1}{\rho_{s} \omega^{2}} \sum_{n=-\infty}^{+\infty} A_{n}^{L} J_{n}\left(k_{L} \rho\right) e^{i n \theta}, \quad \psi=\frac{1}{\rho_{s} \omega^{2}} \sum_{n=-\infty}^{+\infty} A_{n}^{T} J_{n}\left(k_{T} \rho\right) e^{i n \theta}
$$

where $A_{n}^{j}$ are unknown coefficients with $j \in\{L, T\}$. Finally, from the Helmholtz decomposition Eq. (6), the elastic displacement reads

$$
\mathbf{u}=u_{\rho} \mathbf{e}_{\rho}+u_{\theta} \mathbf{e}_{\theta}=\left(-\frac{\partial \phi}{\partial \rho}+\frac{1}{\rho} \frac{\partial \psi}{\partial \theta}\right) \mathbf{e}_{\rho}+\left(-\frac{1}{\rho} \frac{\partial \phi}{\partial \theta}-\frac{\partial \psi}{\partial \rho}\right) \mathbf{e}_{\theta} .
$$

\section{B. Symmetry considerations}

We use a method based on group theory ${ }^{20,22,24}$. The infinite elliptical cylinder is invariant under four symmetry transformations:

(i) $E$, the identity transformation $(\theta \rightarrow \theta)$,

(ii) $C_{2}$, the rotation through $\pi$ about the $O z$ axis $(\theta \rightarrow \pi+\theta)$,

(iii) $\sigma_{x}$, the mirror reflection in the plane $O x z(\theta \rightarrow-\theta)$,

(iv) $\sigma_{y}$, the mirror reflection in the plane $O y z(\theta \rightarrow \pi-\theta)$.

These four transformations form a finite group, called $\mathcal{C}_{2 v}$, which is the symmetry group of the infinite elliptical cylinder ${ }^{23}$. The action of these transformations on the basis vectors $\mathbf{e}_{\rho}$ and $\mathbf{e}_{\theta}$ is given by

$$
\begin{array}{llll}
E\left(\mathbf{e}_{\rho}\right)=\mathbf{e}_{\rho} ; & C_{2}\left(\mathbf{e}_{\rho}\right)=\mathbf{e}_{\rho} ; & \sigma_{x}\left(\mathbf{e}_{\rho}\right)=\mathbf{e}_{\rho} ; & \sigma_{y}\left(\mathbf{e}_{\rho}\right)=\mathbf{e}_{\rho} \\
E\left(\mathbf{e}_{\theta}\right)=\mathbf{e}_{\theta} ; & C_{2}\left(\mathbf{e}_{\theta}\right)=\mathbf{e}_{\theta} ; & \sigma_{x}\left(\mathbf{e}_{\theta}\right)=-\mathbf{e}_{\theta} ; & \sigma_{y}\left(\mathbf{e}_{\theta}\right)=-\mathbf{e}_{\theta}
\end{array}
$$

and, on any scalar function $f(\rho, \theta)$,

$E f(\rho, \theta)=f(\rho, \theta), \quad C_{2} f(\rho, \theta)=f(\rho, \pi+\theta), \quad \sigma_{x} f(\rho, \theta)=f(\rho,-\theta), \quad \sigma_{y} f(\rho, \theta)=f(\rho, \pi-\theta)$. 
TABLE I. Character table of $\mathcal{C}_{2 v}$.

\begin{tabular}{ccccc}
\hline \hline $\mathcal{C}_{2 v}:$ & $E$ & $C_{2}$ & $\sigma_{x}$ & $\sigma_{y}$ \\
\hline$A_{1}$ & 1 & 1 & 1 & 1 \\
$A_{2}$ & 1 & 1 & -1 & -1 \\
$B_{1}$ & 1 & -1 & 1 & -1 \\
$B_{2}$ & 1 & -1 & -1 & 1 \\
\hline \hline
\end{tabular}

Four one-dimensional irreducible representations labelled $A_{1}, A_{2}, B_{1}, B_{2}$ are associated with this symmetry group $\mathcal{C}_{2 v}$. In a given representation, the group elements $E, C_{2}, \sigma_{x}$ and $\sigma_{y}$ are represented by $1 \times 1$ matrices given in the corresponding row of the character table (Table I) .

Following the method presented in Ref. 24, any scalar function $f(\rho, \theta)$ can be expressed as a sum of functions belonging to the four irreducible representations of $\mathcal{C}_{2 v}$ :

$$
f(\rho, \theta)=f^{A_{1}}(\rho, \theta)+f^{A_{2}}(\rho, \theta)+f^{B_{1}}(\rho, \theta)+f^{B_{2}}(\rho, \theta),
$$

where $f^{R_{i}}, R_{i} \in\left\{A_{1}, A_{2}, B_{1}, B_{2}\right\}$, satisfy

$$
\begin{aligned}
f^{A_{1}} & =\frac{1}{4}\left(E+C_{2}+\sigma_{x}+\sigma_{y}\right) f \\
f^{A_{2}} & =\frac{1}{4}\left(E+C_{2}-\sigma_{x}-\sigma_{y}\right) f \\
f^{B_{1}} & =\frac{1}{4}\left(E-C_{2}+\sigma_{x}-\sigma_{y}\right) f \\
f^{B_{2}} & =\frac{1}{4}\left(E-C_{2}-\sigma_{x}+\sigma_{y}\right) f .
\end{aligned}
$$

More recently ${ }^{22}$, in the vectorial context of elastodynamics, it has been shown that any vectorial function, expressed in the polar coordinates system as $\mathbf{f}(\rho, \theta)=f_{\rho} \mathbf{e}_{\rho}+f_{\theta} \mathbf{e}_{\theta}$ can be split over the four irreducible representations of $\mathcal{C}_{2 v}$ :

$$
\mathbf{f}(\rho, \theta)=\mathbf{f}^{A_{1}}(\rho, \theta)+\mathbf{f}^{A_{2}}(\rho, \theta)+\mathbf{f}^{B_{1}}(\rho, \theta)+\mathbf{f}^{B_{2}}(\rho, \theta)
$$

with

$$
\begin{aligned}
\mathbf{f}^{A_{1}} & =f_{\rho}^{A_{1}} \mathbf{e}_{\rho}+f_{\theta}^{A_{2}} \mathbf{e}_{\theta} \\
\mathbf{f}^{A_{2}} & =f_{\rho}^{A_{2}} \mathbf{e}_{\rho}+f_{\theta}^{A_{1}} \mathbf{e}_{\theta} \\
\mathbf{f}^{B_{1}} & =f_{\rho}^{B_{1}} \mathbf{e}_{\rho}+f_{\theta}^{B_{2}} \mathbf{e}_{\theta} \\
\mathbf{f}^{B_{2}} & =f_{\rho}^{B_{2}} \mathbf{e}_{\rho}+f_{\theta}^{B_{1}} \mathbf{e}_{\theta} .
\end{aligned}
$$


TABLE II. Multiplication table for a function belonging to a given irreducible representation.

\begin{tabular}{ccccc}
\hline \hline$\times$ & $A_{1}$ & $A_{2}$ & $B_{1}$ & $B_{2}$ \\
\hline$A_{1}$ & $A_{1}$ & $A_{2}$ & $B_{1}$ & $B_{2}$ \\
$A_{2}$ & $A_{2}$ & $A_{1}$ & $B_{2}$ & $B_{1}$ \\
$B_{1}$ & $B_{1}$ & $B_{2}$ & $A_{1}$ & $A_{2}$ \\
$B_{2}$ & $B_{2}$ & $B_{1}$ & $A_{2}$ & $A_{1}$ \\
\hline \hline
\end{tabular}

It should be noted that the scalar components of a vector function in a given irreducible representation do not necessarily belong to the same representation (see Eqs. (14)).

In the following, the symmetry decompositions Eqs. (11) and (13) will be applied to both scalar (acoustic pressure) and vectorial functions (elastic displacement) respectively. We will deal with scalar functions of the form (see acoustic pressure Eqs. (4) and (5), longitudinal and transverse fields Eq. (7) )

$$
f(\rho, \theta)=\sum_{n=-\infty}^{+\infty} f_{n}(\rho) e^{i n \theta}
$$

Using the action of the transformations on the function $f(\mathrm{Eq} .(10))$ and $f^{R_{i}}$ given by Eqs. (12), one can write the scalar decomposition (Eq. (11)) with

$$
\begin{aligned}
& f^{\mathrm{A}_{1}}(\rho, \theta)=\frac{1}{4} \sum_{n=-\infty}^{+\infty}\left(1+(-1)^{n}\right)\left(f_{n}(\rho)+f_{-n}(\rho)\right) e^{i n \theta} \\
& f^{\mathrm{A}_{2}}(\rho, \theta)=\frac{1}{4} \sum_{n=-\infty}^{+\infty}\left(1+(-1)^{n}\right)\left(f_{n}(\rho)-f_{-n}(\rho)\right) e^{i n \theta} \\
& f^{\mathrm{B}_{1}}(\rho, \theta)=\frac{1}{4} \sum_{n=-\infty}^{+\infty}\left(1-(-1)^{n}\right)\left(f_{n}(\rho)+f_{-n}(\rho)\right) e^{i n \theta} \\
& f^{\mathrm{B}_{2}}(\rho, \theta)=\frac{1}{4} \sum_{n=-\infty}^{+\infty}\left(1-(-1)^{n}\right)\left(f_{n}(\rho)-f_{-n}(\rho)\right) e^{i n \theta} .
\end{aligned}
$$

Restriction to the fundamental domain The use of group theory allows us to restrict the study to the so-called fundamental domain which reduces to $\theta \in[0, \pi / 2]$ (see Fig. 2). This constitutes a great improvement from both theoretical and numerical point of view. Of course, the physical quantities of interest can be determined for the full domain from simple symmetry considerations (Eqs. (11) and (13)). 


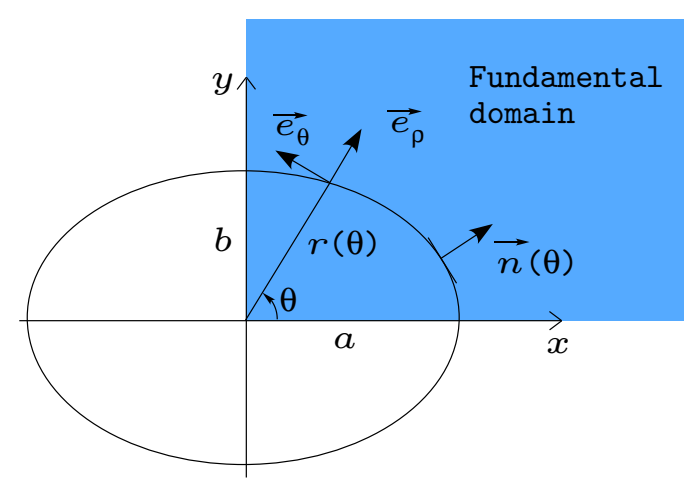

FIG. 2. Fundamental domain.

\section{SOLVING THE SCATTERING PROBLEM}

\section{A. Boundary conditions}

In order to determined the resonances and the unknown coefficients, the boundary conditions

(i) Continuity of the normal displacement $\mathbf{u} \cdot \mathbf{n}(\theta)=\frac{1}{\rho_{f} \omega^{2}} \nabla\left(p_{\text {inc }}+p_{s}\right) \cdot \mathbf{n}(\theta)$

(ii) Continuity of the stress $\mathbf{t}=\overline{\bar{\sigma}} \cdot \mathbf{n}(\theta)=-\left(p_{\text {inc }}+p_{s}\right) \mathbf{n}(\theta)$

are applied at the surface $\rho=r(\theta)$ of the scatterer. According to the expressions of $\mathbf{n}(\theta)$, $u_{\rho}$ and $u_{\theta}$ given by Eqs. (1) and (8) respectively, they read

(i)

$$
\left(-\frac{\partial \phi}{\partial \rho}+\frac{1}{\rho} \frac{\partial \psi}{\partial \theta}\right) n_{\rho}+\left(-\frac{1}{\rho} \frac{\partial \phi}{\partial \theta}-\frac{\partial \psi}{\partial \rho}\right) n_{\theta}=\frac{1}{\rho_{f} \omega^{2}}\left(\frac{\partial\left(p_{i n c}+p_{s}\right)}{\partial \rho} n_{\rho}+\frac{1}{\rho} \frac{\partial\left(p_{i n c}+p_{s}\right)}{\partial \theta} n_{\theta}\right)
$$

with $p_{i n c}$ and $p_{s}$ given by Eqs. (4) and (5) respectively.

(ii)

$$
t_{\rho} \mathbf{e}_{\rho}+t_{\theta} \mathbf{e}_{\theta}=\left(\sigma_{\rho \rho} n_{\rho}+\sigma_{\rho \theta} n_{\theta}\right) \mathbf{e}_{\rho}+\left(\sigma_{\rho \theta} n_{\rho}+\sigma_{\theta \theta} n_{\theta}\right) \mathbf{e}_{\theta}=-\left(p_{i n c}+p_{s}\right)\left(n_{\rho} \mathbf{e}_{\rho}+n_{\theta} \mathbf{e}_{\theta}\right)
$$

i.e.,

$$
\begin{aligned}
\sigma_{\rho \rho} n_{\rho}+\sigma_{\rho \theta} n_{\theta} & =-\left(p_{i n c}+p_{s}\right) n_{\rho} \\
\sigma_{\rho \theta} n_{\rho}+\sigma_{\theta \theta} n_{\theta} & =-\left(p_{i n c}+p_{s}\right) n_{\theta}
\end{aligned}
$$


The components of $\overline{\bar{\sigma}}$ in circular cylindrical coordinates are obtained from the strain tensor $^{26}$, Hooke's law, and the Helmholtz equation for the scalar potential $\phi$

$$
\begin{aligned}
& \sigma_{\rho \rho}=\lambda k_{L}^{2} \phi-2 \mu\left(\frac{\partial^{2} \phi}{\partial \rho^{2}}+\frac{1}{\rho^{2}} \frac{\partial \psi}{\partial \theta}-\frac{1}{\rho} \frac{\partial^{2} \psi}{\partial \rho \partial \theta}\right) \\
& \sigma_{\rho \theta}=\sigma_{\theta \rho}=\mu\left(-\frac{2}{\rho} \frac{\partial^{2} \phi}{\partial \theta \partial \rho}+\frac{1}{\rho^{2}} \frac{\partial^{2} \psi}{\partial \theta^{2}}+\frac{1}{\rho} \frac{\partial \psi}{\partial \rho}-\frac{\partial^{2} \psi}{\partial \rho^{2}}+\frac{2}{\rho^{2}} \frac{\partial \phi}{\partial \theta}\right) \\
& \sigma_{\theta \theta}=\lambda k_{L}^{2} \phi+2 \mu\left(-\frac{1}{\rho^{2}} \frac{\partial^{2} \phi}{\partial \theta^{2}}-\frac{1}{\rho} \frac{\partial^{2} \psi}{\partial \theta \partial \rho}-\frac{1}{\rho} \frac{\partial \phi}{\partial \rho}+\frac{1}{\rho^{2}} \frac{\partial \psi}{\partial \theta}\right) .
\end{aligned}
$$

The boundary conditions (i) and (ii) can now be expressed separately in each irreducible representation. We note that $\mathbf{n}(\theta)$ defined by Eq. (1) belongs to $A_{1}$ for obvious symmetry considerations, thus its components $n_{\rho}$ and $n_{\theta}$ respectively belong to $A_{1}$ and $A_{2}$ under the property Eq. (14a). Then, we use the latter property, the multiplication table (Table II) and we note that the derivative by respect to $\theta$ acts as a multiplication by some function belonging to the representation $A_{2}$. In order to write Eqs. (17) and (18) in each irreducible representation $R_{i}$, one deduces from these rules, that $\phi, p_{s}$ and $p_{i n c}$ have to belong to $R_{1}$ (resp. $R_{2}$ ) while $\psi$ has to belong to $R_{2}$ (resp. $R_{1}$ ). Here, $R$ stands for $A$ or $B$ exclusively.

In what follows, the infinite sums appearing in Eqs. (4), (5) and (7) are reduced using Eqs. (16) and for clarity, we define

$$
\sum_{n \text { even }} f_{n}=\sum_{r=0}^{+\infty} f_{2 r}, \quad \text { and } \quad \sum_{n \text { odd }} f_{n}=\sum_{r=0}^{+\infty} f_{2 r+1}
$$

Moreover, the unknown coefficients $A_{n}^{\left(R_{i}\right) L}, A_{n}^{\left(R_{i}\right) T}, A_{n}^{\left(R_{i}\right) S}$ are respectively written $A_{n}^{L}, A_{n}^{T}$, $A_{n}^{S}$, their dependence to a given irreducible representation is implicit.

Solving the scattering problem requires to overcome the angular dependence. Then, the expressions involving $r(\theta)$ and $\boldsymbol{n}(\theta)$ are expanded in Fourier series by setting

$$
F_{n}(\theta)=\sum_{p=-\infty}^{+\infty} f_{n, p} e^{i p \theta}, \quad \text { with } f_{n, p}=\frac{1}{2 \pi} \int_{-\pi}^{\pi} F_{n}(\theta) e^{-i p \theta} d \theta
$$

In Eq. (22), the restriction to the fundamental domain and the parity of $F_{n}(\theta)$ permits one to reduce the domain of integration from $[-\pi, \pi]$ to $[0, \pi / 2]$ and the sum over $p$ from $[-\infty,+\infty]$ to $[0,+\infty]$ with $p$ even $\left(A_{1}, A_{2}\right)$ or $p$ odd $\left(B_{1}, B_{2}\right)$. Then, we obtain, for each 
irreducible representation, a system of equations of the form

$$
\begin{aligned}
& \sum_{n, p} \gamma_{n} \gamma_{p}\left[A_{n}^{L} \mathcal{M}_{n, p}^{L}+A_{n}^{T} \mathcal{M}_{n, p}^{T}+A_{n}^{S} \mathcal{M}_{n, p}^{S}\right] \begin{array}{l}
\cos p \theta \\
\sin p \theta
\end{array}=\sum_{n, p} \gamma_{n} \gamma_{p} \mathcal{M}_{n, p}^{i n c} \cos n \alpha \cos p \theta\left(A_{1}, B_{1}\right) \\
& \sum_{n, p} \gamma_{n} \gamma_{p}\left[A_{n}^{L} \mathcal{N}_{n, p}^{L}+A_{n}^{T} \mathcal{N}_{n, p}^{T}+A_{n}^{S} \mathcal{N}_{n, p}^{S}\right] \begin{array}{l}
\cos p \theta \\
\sin p \theta
\end{array}=\sum_{n, p} \gamma_{n} \gamma_{p} \mathcal{N}_{n, p}^{i n c} \cos n \alpha \cos p \theta \quad\left(A_{1}, B_{1}\right)
\end{aligned}
$$

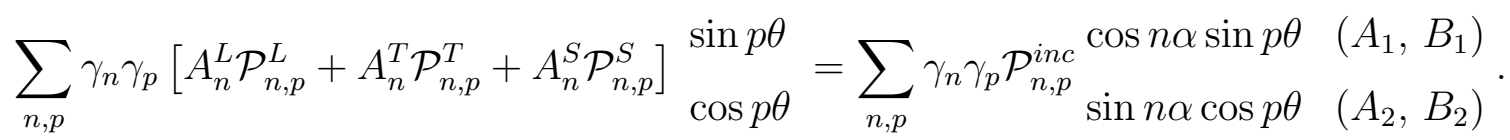

where $\gamma_{i}, i \in\{n, p\}$, is the Neuman factor defined by $\gamma_{0}=1$ and $\gamma_{i}=2$ for $n>0$. The matrices $\mathcal{M}_{n, p}^{j}, \mathcal{N}_{n, p}^{j}$ and $\mathcal{P}_{n, p}^{j}, j \in\{L, T, S, i n c\}$, are the Fourier coefficients. The detailed calculations for each irreducible representation are given in Appendix A.

\section{B. Physical quantities of interest}

The scattering of a plane acoustic wave by an infinite elastic cylinder of elliptical cross section reduces to the solution of equations Eqs. (A5), (A9), (A13), (A17), an infinite set of infinite systems of linear complex algebraic equations. Each system is associated with a given irreducible representation of $\mathcal{C}_{2 v}$. The unknown scattering coefficients are uncoupled due to symmetry considerations, this greatly simplifies the treatment of the problem. Then the systems of equations can be numerically solved by truncation and used to obtain i) the resonances, ii) the elastic displacement, iii) the scattered pressure and iv) the far field form function.

\section{Determination of resonances}

The resonances are determined by solving the characteristic equation for each representation $R_{i}$

$$
\operatorname{det} M^{R_{i}}=0
$$

in the complex $k r$-plane, where $k r$ is the reduced wave number in the fluid ${ }^{27}$, and they are naturally classified according to the irreducible representations $R_{i} . M^{R_{i}}$ is the matrix obtained from the systems of equations Eqs. (A5), (A9), (A13) and (A17). 


\section{Elastic displacement}

The elastic displacement Eq. (8) can be expressed in each irreducible representation $R_{i}$ using the vectorial decompositions Eqs. (14) and the potentials Eqs. (A1), (A6), (A10) and (A14). Then we get

$$
\begin{aligned}
& \mathbf{u}^{A_{1}}=\frac{1}{\rho_{s} \omega^{2}} \sum_{n \text { even }} \gamma_{n} {\left[\left(-k_{L} A_{n}^{L} J_{n}^{\prime}\left(k_{L} \rho\right)+i \frac{n}{\rho} A_{n}^{T} J_{n}\left(k_{T} \rho\right)\right) \cos (n \theta) \mathbf{e}_{\rho}\right.} \\
&+\left.\left(-i k_{T} A_{n}^{T} J_{n}^{\prime}\left(k_{T} \rho\right)+\frac{n}{\rho} A_{n}^{L} J_{n}\left(k_{L} \rho\right)\right) \sin (n \theta) \mathbf{e}_{\theta}\right] \\
& \mathbf{u}^{A_{2}}=\frac{1}{\rho_{s} \omega^{2}} \sum_{n \text { even }} \gamma_{n}\left[\left(-i k_{L} A_{n}^{L} J_{n}^{\prime}\left(k_{L} \rho\right)-\frac{n}{\rho} A_{n}^{T} J_{n}\left(k_{T} \rho\right)\right) \sin (n \theta) \mathbf{e}_{\rho}\right. \\
&\left.+\left(-k_{T} A_{n}^{T} J_{n}^{\prime}\left(k_{T} \rho\right)-i \frac{n}{\rho} A_{n}^{L} J_{n}\left(k_{L} \rho\right)\right) \cos (n \theta) \mathbf{e}_{\theta}\right] \\
& \mathbf{u}^{B_{1}}=\frac{2}{\rho_{s} \omega^{2}} \sum_{n \text { odd }} {\left[\left(-k_{L} A_{n}^{L} J_{n}^{\prime}\left(k_{L} \rho\right)+i \frac{n}{\rho} A_{n}^{T} J_{n}\left(k_{T} \rho\right)\right) \cos (n \theta) \mathbf{e}_{\rho}\right.} \\
&+\left.\left(-i k_{T} A_{n}^{T} J_{n}^{\prime}\left(k_{T} \rho\right)+\frac{n}{\rho} A_{n}^{L} J_{n}\left(k_{L} \rho\right)\right) \sin (n \theta) \mathbf{e}_{\theta}\right] \\
&+\left.\left(-k_{T} A_{n}^{T} J_{n}^{\prime}\left(k_{T} \rho\right)-i \frac{n}{\rho} A_{n}^{L} J_{n}\left(k_{L} \rho\right)\right) \cos (n \theta) \mathbf{e}_{\theta}\right] \\
& \mathbf{u}^{B_{2}}=\frac{2}{\rho_{s} \omega^{2}} \sum_{n \text { odd }} {\left[\left(-i k_{L} A_{n}^{L} J_{n}^{\prime}\left(k_{L} \rho\right)-\frac{n}{\rho} A_{n}^{T} J_{n}\left(k_{T} \rho\right)\right) \sin (n \theta) \mathbf{e}_{\rho}\right.} \\
&+
\end{aligned}
$$

The displacement normal modes can then be obtained by computing $\left|\mathbf{u}^{R_{i}}\right|$.

\section{Scattered pressure and Far field form function}

The scattered pressure Eq. (5) can be expressed in each irreducible representation $R_{i}$ using $p_{s}^{R_{i}}$ given by Eqs. (A2), (A7), (A11) and (A15).

The form function $F F_{\infty}$ is defined by

$$
F F_{\infty}=\lim _{r \rightarrow \infty} \sqrt{\frac{a_{\text {eff }}}{a}} \sqrt{\frac{2 \rho}{a}}\left|\frac{p_{s}}{p_{\text {inc }}}\right|
$$


with $a_{\mathrm{eff}}=\sqrt{\frac{a^{2}+b^{2}}{2}}$ and $p_{s}=p_{s}^{\mathrm{A}_{1}}+p_{s}^{\mathrm{A}_{2}}+p_{s}^{\mathrm{B}_{1}}+p_{s}^{\mathrm{B}_{2}}$ obtained from the scalar decomposition Eq. (11). Equivalently, by taking the limit of each component of the scattered pressure, one obtains.

$$
F F_{\infty}=\left|F F_{\infty}^{A_{1}}+F F_{\infty}^{A_{2}}+F F_{\infty}^{B_{1}}+F F_{\infty}^{B_{2}}\right|
$$

with

$$
\begin{gathered}
F F_{\infty}^{A_{1}}=\frac{2}{a} \sqrt{\frac{a_{\text {eff }}}{\pi k}} e^{-i \pi / 4} \sum_{n \text { even }} \gamma_{n} A_{n}^{S} \cos (n \theta) \\
F F_{\infty}^{A_{2}}=\frac{2 i}{a} \sqrt{\frac{a_{\text {eff }}}{\pi k}} e^{-i \pi / 4} \sum_{n \text { even }} \gamma_{n} A_{n}^{S} \sin (n \theta) \\
F F_{\infty}^{B_{1}}=\frac{4 i}{a} \sqrt{\frac{a_{\text {eff }}}{\pi k}} e^{-3 i \pi / 4} \sum_{n \text { odd }} A_{n}^{S} \cos (n \theta) \\
F F_{\infty}^{B_{2}}=-\frac{4}{a} \sqrt{\frac{a_{\text {eff }}}{\pi k}} e^{-3 i \pi / 4} \sum_{n \text { odd }} A_{n}^{S} \sin (n \theta) .
\end{gathered}
$$

\section{NUMERICAL RESULTS AND COMPARISON WITH EXPERIMENT}

\section{A. Numerical considerations}

The numerical determination of the physical quantities described above (except for the resonances) requires the evaluation of the unknown coefficients $A_{n}^{L}, A_{n}^{T}, A_{n}^{S}$ for each irreducible representation $R_{i}$. These coefficients are obtained by solving the truncated complex linear systems Eqs. (A5), (A9), (A13), (A17). Regarding the resonances, we recall that they are determined by solving the characteristic equation Eq. (24). Since the matrices involved in these computations are of infinite dimensionality, some truncation order $N$ must be performed in order to generate a numerical solution. The chosen truncation order depends on the dimensionless reduced wave number $k a$ ( $a$ is the semi-major axis of the elliptical cylinder) and it has been numerically investigated. With the help of group theory, the matrices involved in our problem are of dimension $(N \times N)$. This constitutes a great improvement compared with the coupled problem (without group theory) that involves $(2 N \times 2 N)$ matrices.

Further the numerical enhancements, the main advantage of group theory is to obtain uncoupled equations that can be solved separately for each irreducible representation. This leads to a full classification of the resonances and this provides consequently a physical 
TABLE III. Characteristics of the transducers.

\begin{tabular}{cccc}
\hline \hline & Central frequency & Bandwith $(-12 \mathrm{~dB})$ & Radiating surface diameter \\
\hline V389 & $f \approx 475 \mathrm{kHz}$ & $250 \mathrm{kHz} \lesssim f \lesssim 700 \mathrm{kHz}$ & $4 \mathrm{~cm}$ \\
& $k r \approx 15$ & $8 \lesssim k r \lesssim 22$ & \\
\hline V392 & $f \approx 870 \mathrm{kHz}$ & $400 \mathrm{kHz} \lesssim f \lesssim 1300 \mathrm{kHz}$ & $4 \mathrm{~cm}$ \\
& $k r \approx 28$ & $13 \lesssim k r \lesssim 41$ & \\
\hline \hline
\end{tabular}

interpretation in terms of symmetry. Furthermore, computations can also be carried out at high frequency.

\section{B. Experimental set-up}

A series of experiments have been performed in the case of scattering of acoustic waves from aluminium Ag4mc ( $\left.\rho_{s}=2670 \mathrm{~kg} . \mathrm{m}^{-3} ; c_{L}=6277 \mathrm{~m} . \mathrm{s}^{-1} ; c_{T}=3162 \mathrm{~m} . \mathrm{s}^{-1}\right)$ elliptical cylinders of length $40 \mathrm{~cm}$ and for various ratios $b / a(0.9640-0.9051-0.7616-0.6351-0.5370)$ immersed in a water tank $\left(190 \mathrm{~cm} \times 130 \mathrm{~cm} \times 90 \mathrm{~cm}-\rho_{w}=1000 \mathrm{~kg} \cdot \mathrm{m}^{-3}, c_{w}=1482.7 \mathrm{~m} \cdot \mathrm{s}^{-1}\right)$. It should be noted that the cylinders are machined at constant perimeter in order to keep an identical travel time for any circumferential wave, whatever the ratio $b / a$. The transducers are PANAMETRICS models whose characteristics are summarized in the table III. The distance between the cylinders and the transducers is $60 \mathrm{~cm}$; it is sufficient to ensure the far field condition.

The experimental results are obtained by ultrasonic spectroscopy. Two types of measurements are performed:

a. Monostatic set-up The experimental form functions have been carried out in the monostatic configuration. The ultrasonic transducers are used both for emission and reception (backscattering). This method consists of using short ultrasonic pulses for excitation and calculating the Fourier transform of the gated-averaged signal reflected from the elliptical cylinders. The received signal is amplified, averaged, sampled and stored in order to perform further calculations by Fast Fourier Transform. The amplitude spectra measured are then normalized by those obtained form a perfectly reflecting surface (water surface). This permits one to overcome the frequency response of the transducers. By this way, the 
theoretical and experimental results can be directly compared.

b. Bistatic set-up The experimental bistatic acoustic patterns (angular diagrams) have been carried out in the bistatic configuration. Two transducers are used, one acts as an emitter and the other one as a receiver. The incident angle is fixed, while the recording angle turns around the cylinder. A sinusoidal pulse of fixed frequency is sent to the emitter. The duration of this pulse has to be large enough to ensure the establishment of a steady state in the scatterer while avoiding interferences between the emitted and the scattered signals. An amplitude measurement is performed in the free response of the scatterer once the emission has been stopped.

Plane wave insonation and symmetry considerations The scatterer is insonified by an acoustic plane wave under various angles of incidence $\alpha$. According to the value of $\alpha$, the total pressure $p=p_{\text {inc }}+p_{s}$ is not invariant under all the symmetry transformations given in section II B and summarized in the character table I.

- $\alpha=45^{\circ}$ (oblique incidence): $p$ only satisfies the identity $E$. This way, we obtain the resonances and the scattered pressure in all the irreducible representations.

- $\alpha=90^{\circ}$ (minor axis incidence): $p$ only satisfies the identity $E$ and the mirror reflection $\sigma_{y}$. Then, we obtain the resonances and the scattered pressure in the two irreducible representations $A_{1}$ and $B_{2}$.

- $\alpha=0^{\circ}$ (major axis incidence): $p$ only satisfies the identity $E$ and the mirror reflection $\sigma_{x}$. Then, we obtain the resonances and the scattered pressure in the two irreducible representations $A_{1}$ and $B_{1}$.

\section{Comparison between numerical and experimental results}

The experimental form functions are compared with the theoretical ones calculated from Eq. (30) on Figs. (3-9). A very good agreement between theory and experiment is observed in the operating range of the transducers (Table III) and beyond ${ }^{28}$. The numerous rapid variations of sharp characteristic shape, predicted theoretically, are observed experimentally. They are associated with the elastic resonances plotted in the complex $k r$-plane. As the 
problem is uncoupled over the 4 irreducible representations of $\mathcal{C}_{2 v}$, a full classification of these resonances is provided.

We focus now on the splitting up of resonances that can be interpreted in terms of symmetry breaking. The circular cylinder symmetry is broken by selecting various elliptical cylinders of decreasing ratio $b / a$. The resonances of the circular cylinder are associated with resonant modes labelled by $(n, \ell)$ in the usual modal formalism. They are tracked as it is deformed to the elliptical one, by keeping the perimeter constant. In order to illustrate the deformation, the considered ellipse and the circle are superimposed in Figs. (3-9).

In Figs. 3 and 4, we emphasize the splitting up of resonances by linking the deep peaks appearing on both theoretical and experimental form functions in oblique incidence (Figs. $3 \mathrm{a}$ and $4 \mathrm{a}$ ) with the resonances located in the complex $k r$-plane ${ }^{29}$ (Figs. 3b and $4 \mathrm{~b}$ ). Among the split modes observed, interest is focused on the mode $(1,3)$ split in $B_{1}$ and $B_{2}$, and the mode $(2,4)$ split in $A_{1}$ and $A_{2}$. It should be noted that both real and imaginary parts of the resonances separates, and

- for any even angular index $n$, the resonances are split in the two irreducible representations $A_{1}$ and $A_{2}$,

- for any odd angular index $n$, the resonances are split in the two irreducible representations $B_{1}$ and $B_{2}$.

Bistatic scattering patterns have been also investigated on Figs. 3c and 4c. The time harmonic frequencies of the plane incident wave are chosen equal to the computed resonance frequencies of the four distinct selected modes. The angle of incidence is fixed following the symmetry considerations described above for the total pressure. The position of the emitting transducer is represented by a plain square symbol on these angular diagrams. The results obtained correspond to the scattered pressure and they permit one to identify unambiguously the modes $(1,3)^{B_{1}},(1,3)^{B_{2}},(2,4)^{A_{1}}$ and $(2,4)^{A_{2}}$. Finally, the computed amplitudes of the elastic displacement and the scattered pressure, for each normal mode, are plotted on Figs. $3 \mathrm{~d}$ and $4 \mathrm{~d}$. The angular diagrams are in a good agreement with the scattered pressure computed for each representation and normalized by the incident pressure.

Once the symmetry is only just broken (See Fig. 3), the splitting up of resonance is clearly observed theoretically and experimentally. Only group theory permits one to highlight this 
phenomenon and to link the split modes with the initial degenerated mode of the circular cylinder.

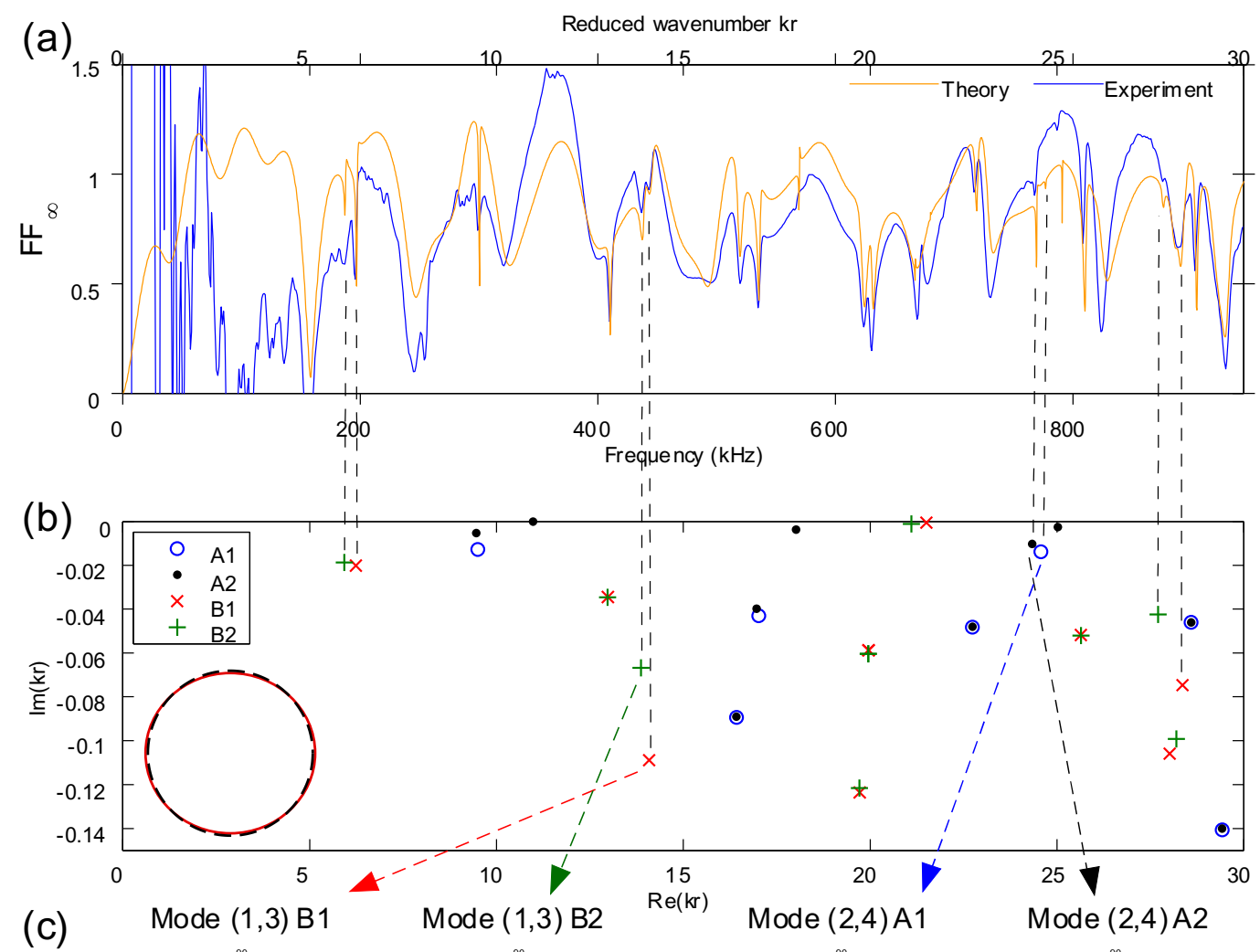

(c)
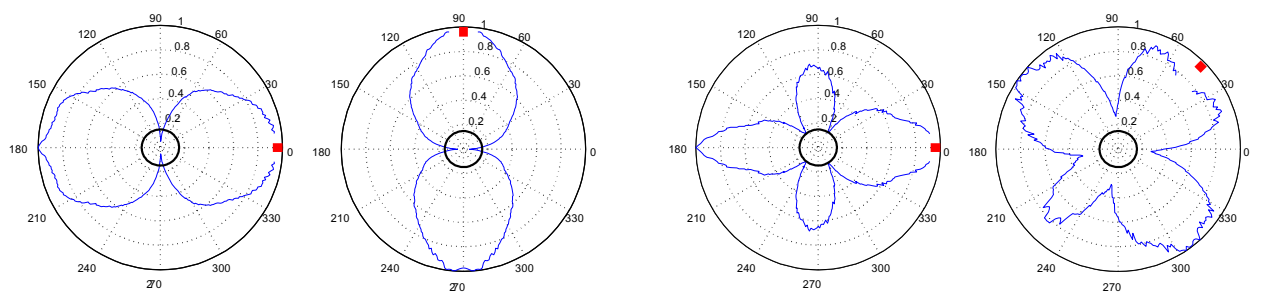

(d)
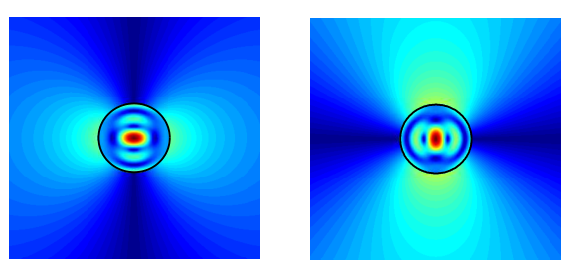

$\mathrm{f}=773 \mathrm{kHz}$

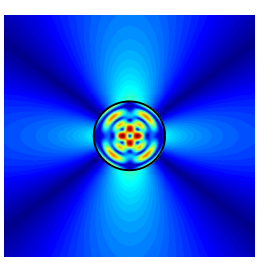

\section{$\mathrm{f}=765.5 \mathrm{kHz}$}

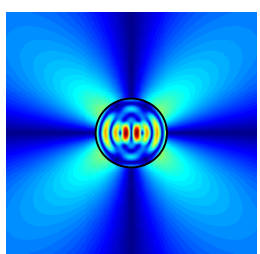

FIG. 3. Elliptical cylinder of ratio $\mathbf{b} / \mathbf{a}=\mathbf{0 . 9 6 4 0}$. (a) Comparison between theoretical and experimental form functions in oblique incidence $\alpha=45^{\circ}$. (b) Location of the computed scattering resonances in the complex $k r$-plane. (c) Experimental bistatic scattering patterns. (d) Computed amplitudes for the elastic displacement and the normalized scattered pressure. 


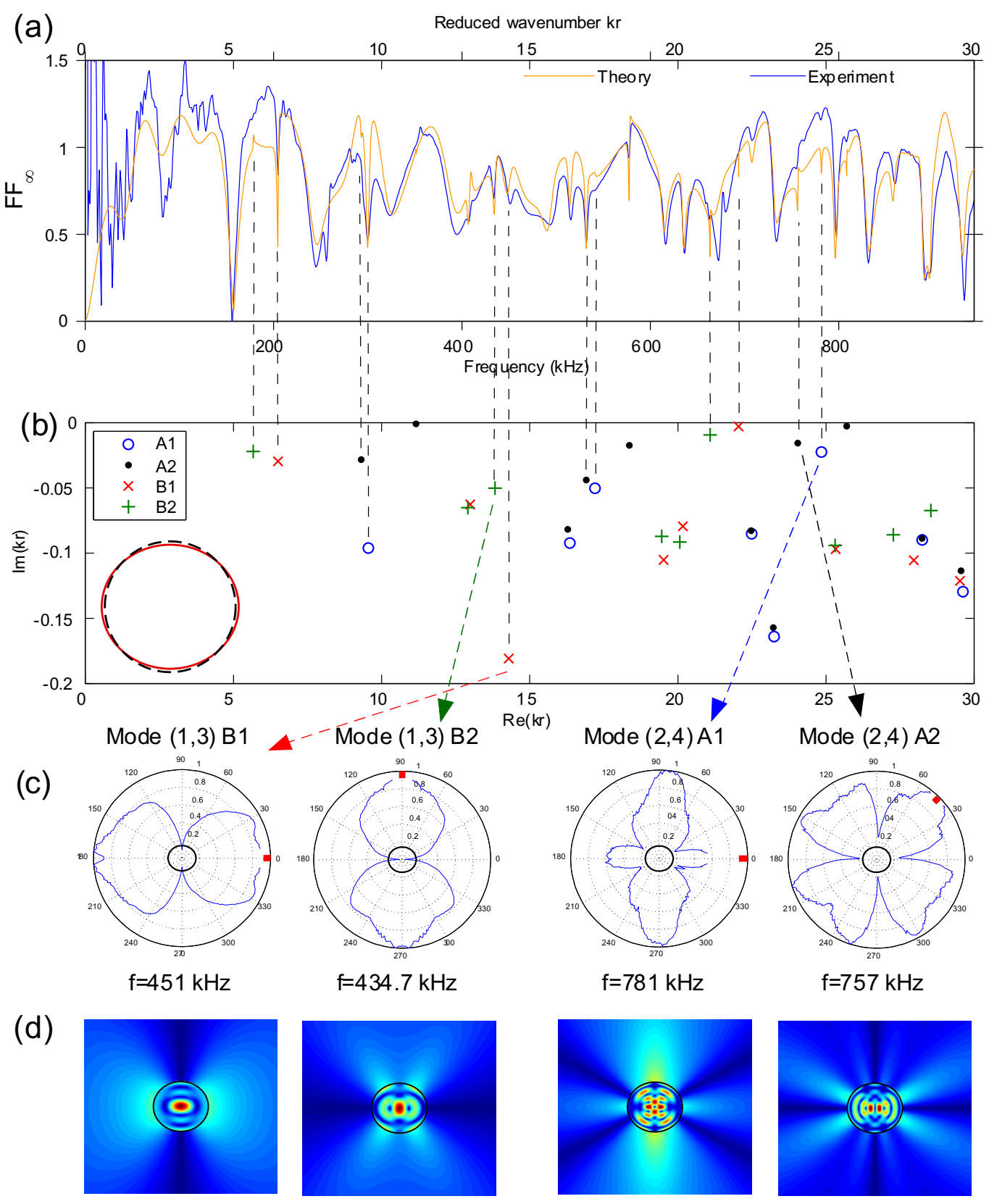

FIG. 4. Elliptical cylinder of ratio $\mathbf{b} / \mathbf{a}=\mathbf{0 . 9 0 5 1}$. (a) Comparison between theoretical and experimental form functions in oblique incidence $\alpha=45^{\circ}$. (b) Location of the computed scattering resonances in the complex $k r$-plane. (c) Experimental bistatic scattering patterns. (d) Computed amplitudes for the elastic displacement and the normalized scattered pressure.

Figs. 5 and 6 show an excellent agreement between experimental and theoretical results, and emphasize the influence of the insonation described in the paragraph related to symmetry considerations of the section IV B . More precisely, 
- on Fig. 5, only the resonances belonging to $A_{1}$ and $B_{2}$ appear on both theoretical and experimental form functions for an angle of incidence equal to $90^{\circ}$,

- on Fig. 6, only the resonances belonging to $A_{1}$ and $B_{1}$ appear on both theoretical and experimental form functions for an angle of incidence equal to $0^{\circ}$.

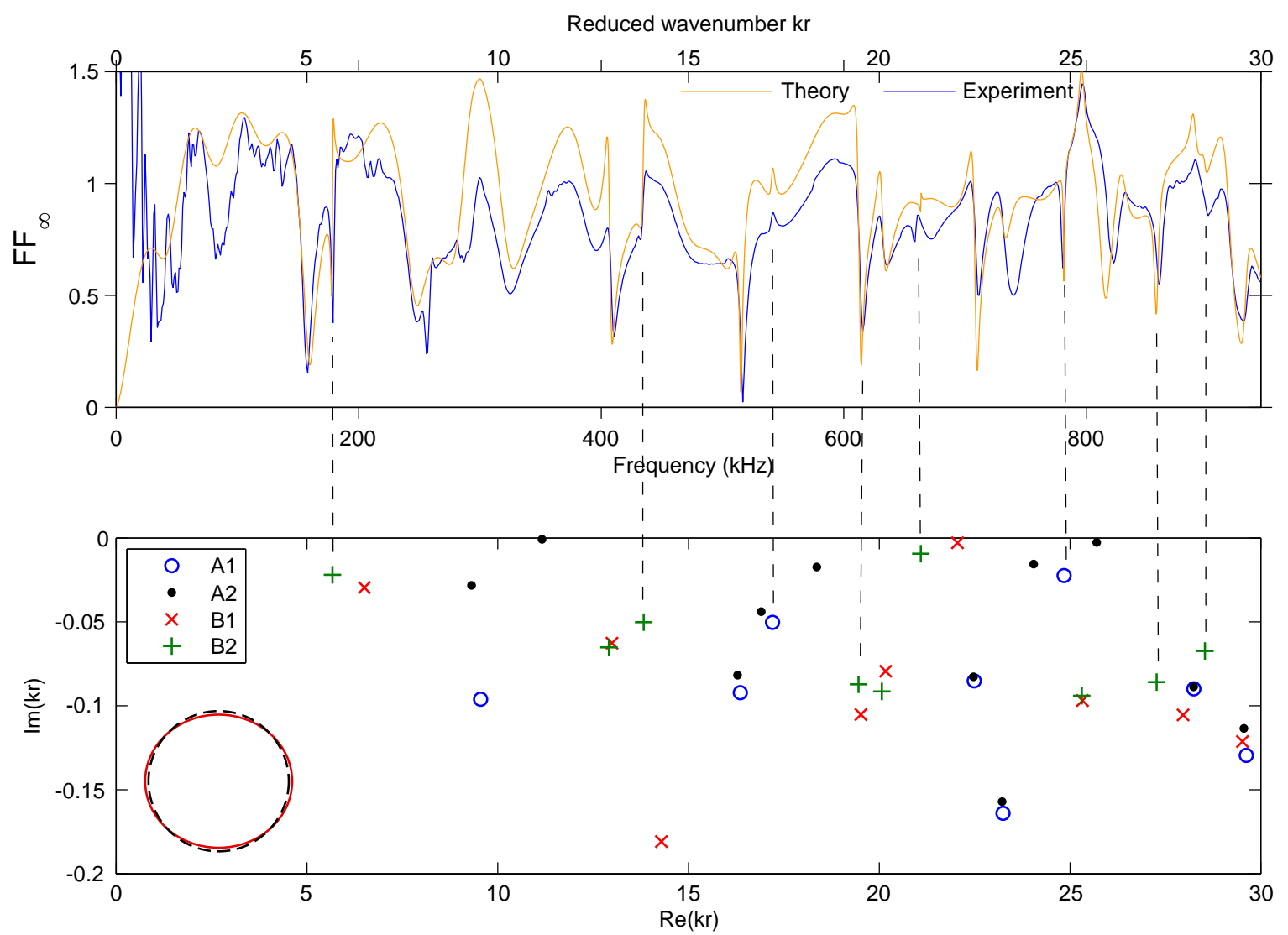

FIG. 5. Elliptical cylinder of ratio $b / a=0.9051$. (Top) Comparison between theoretical and experimental form functions for an angle of incidence equal to $90^{\circ}$ (minor axis). (Bottom) Location of the computed scattering resonances in the complex $k r$-plane. 


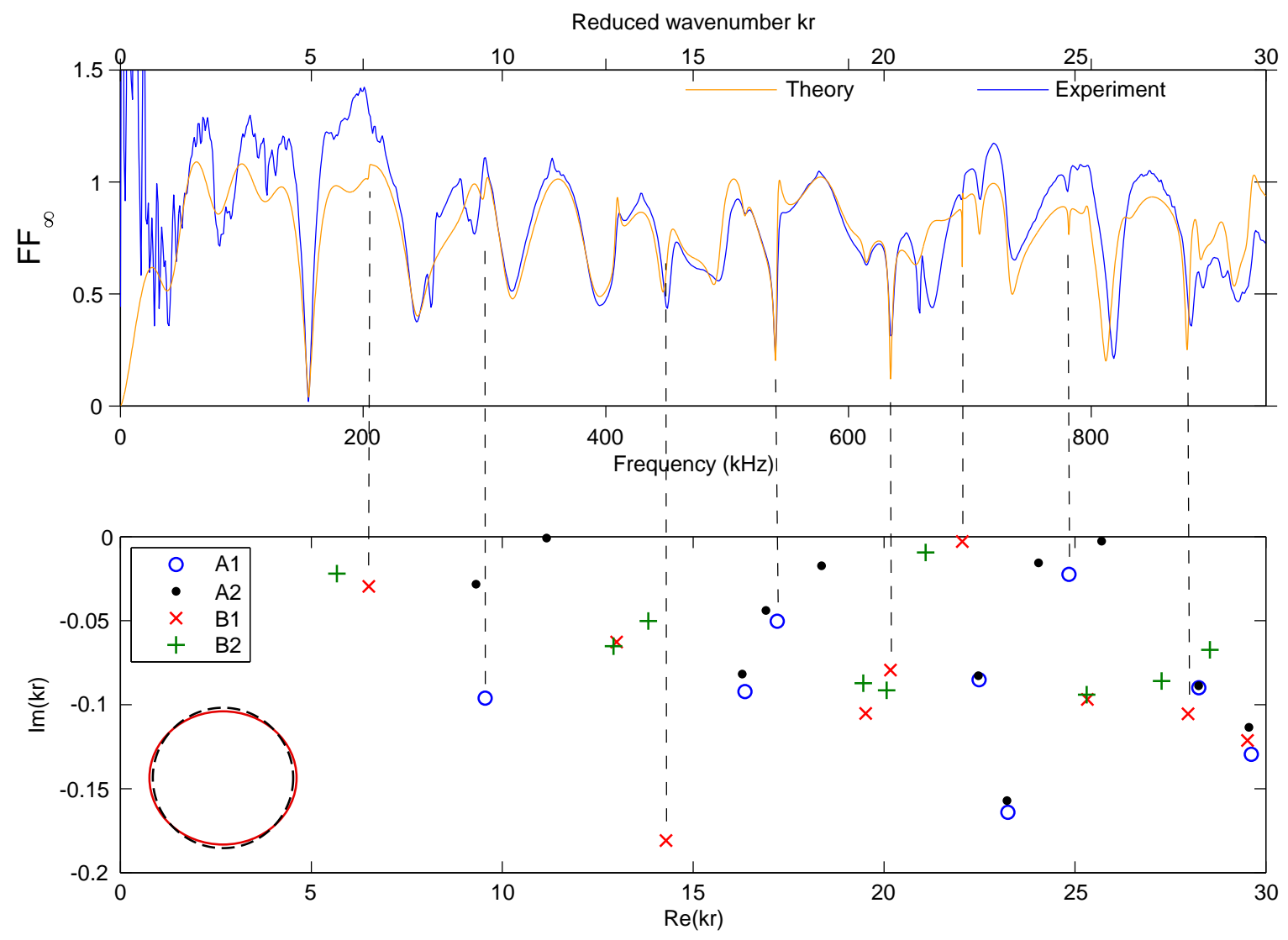

FIG. 6. Elliptical cylinder of ratio $b / a=0.9051$. (Top) Comparison between theoretical and experimental form functions for an angle of incidence equal to $\mathbf{0}^{\circ}$ (major axis). (Bottom) Location of the computed scattering resonances in the complex $k r$-plane.

Figs. 7 and 8 show a very good agreement between theory and experiment for small values of the ratio $b / a$ (large eccentricities). Although the numerical computations to determine the resonances have been also carried out in case of large eccentricities when rapid variations of the curvature radius occur, the identification of modes splitting becomes increasingly difficult. Indeed, numerous resonances are too attenuated to be observed on the form functions since the absolute value of their imaginary part increases.

Finally, a very good agreement is also provided in high frequency domain (up to $k r=50$ ) between experimental and theoretical form functions on Fig. 9. 


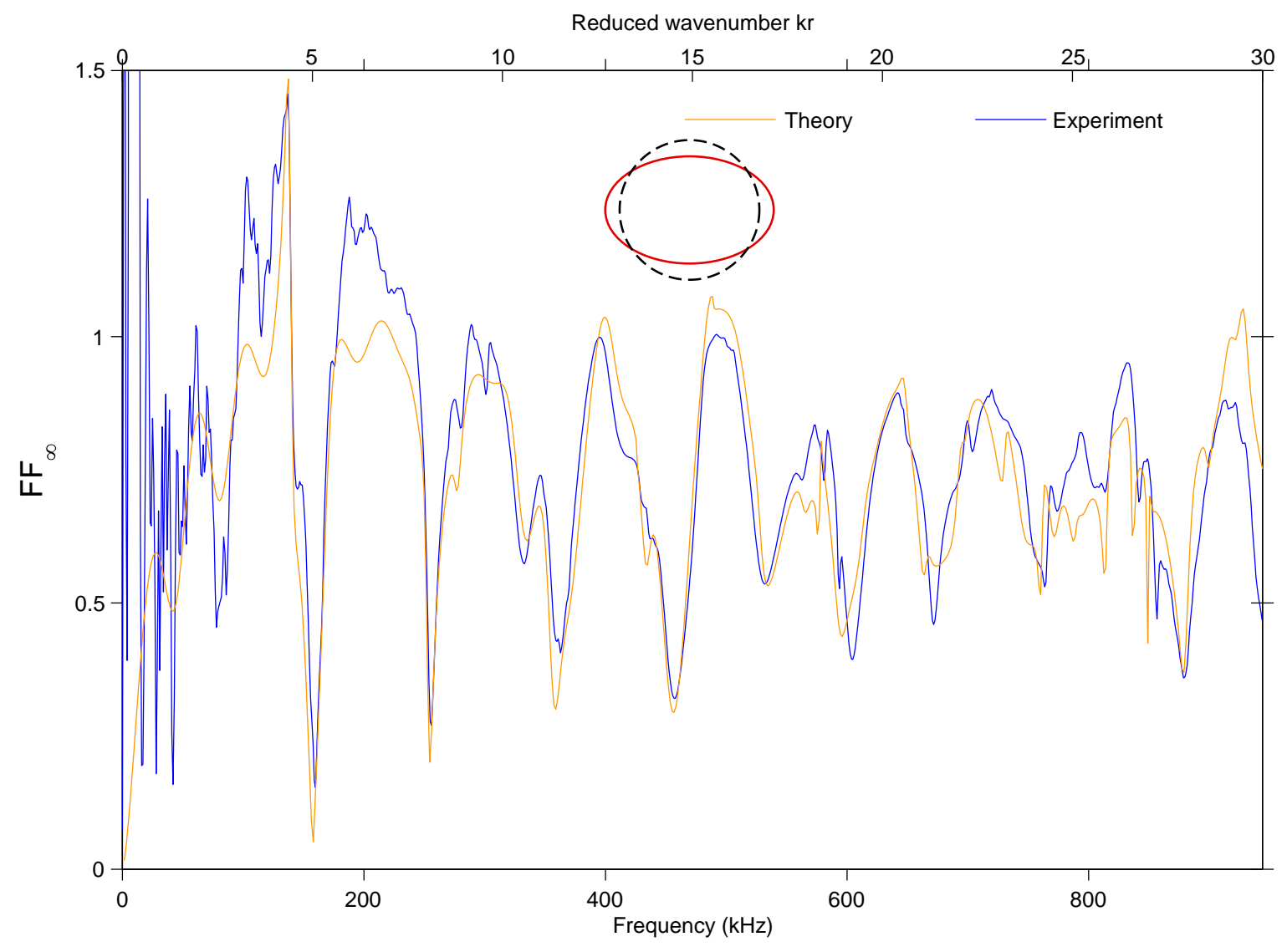

FIG. 7. Comparison between theoretical and experimental form functions for an elliptical cylinder of ratio $\mathbf{b} / \mathbf{a}=\mathbf{0 . 6 3 5 1}$ in oblique incidence $\left(\alpha=45^{\circ}\right)$. 


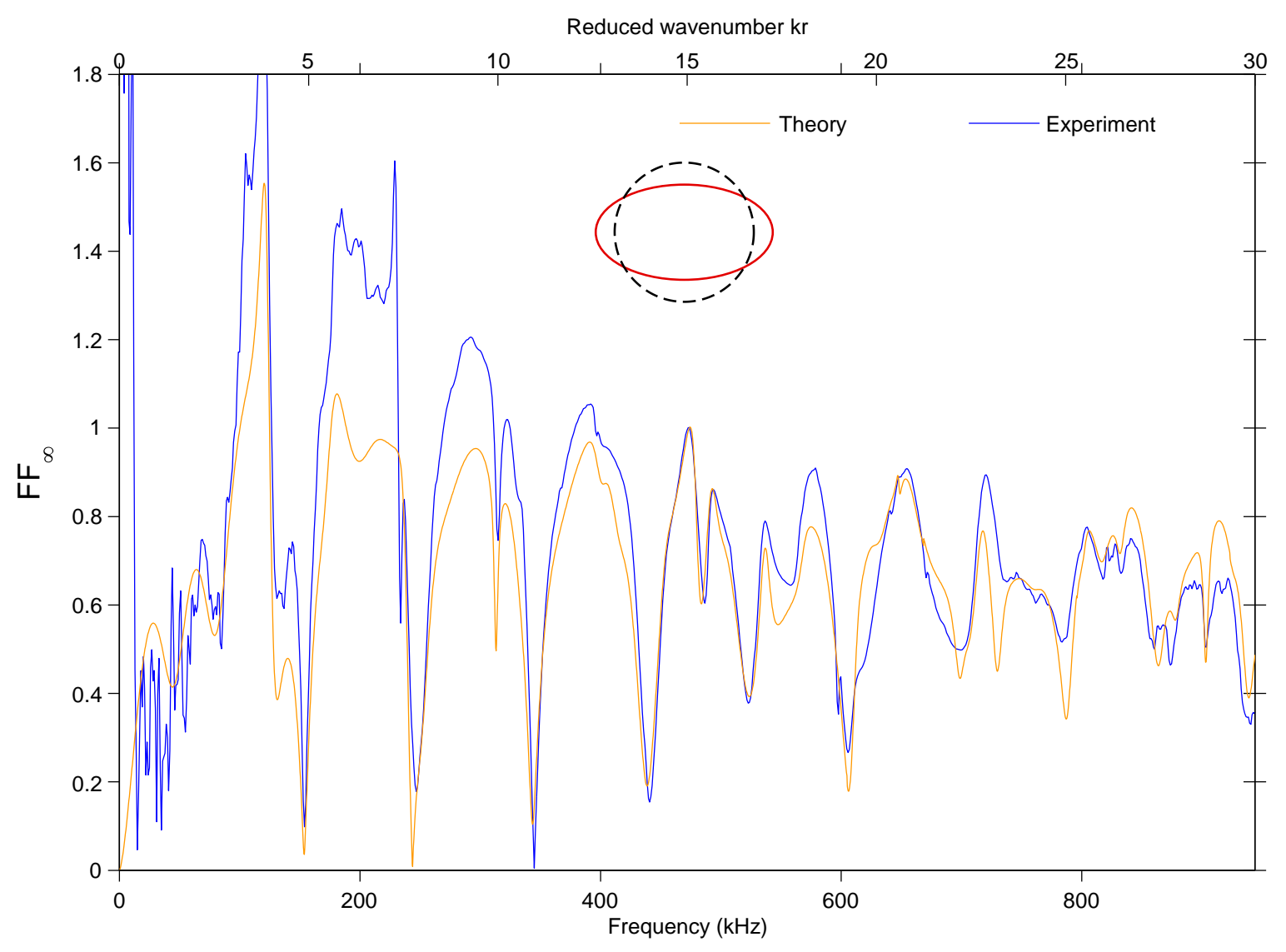

FIG. 8. Comparison between theoretical and experimental form functions for an elliptical cylinder of ratio $\mathbf{b} / \mathbf{a}=\mathbf{0 . 5 3 7 0}$ in oblique incidence $\left(\alpha=45^{\circ}\right)$. 


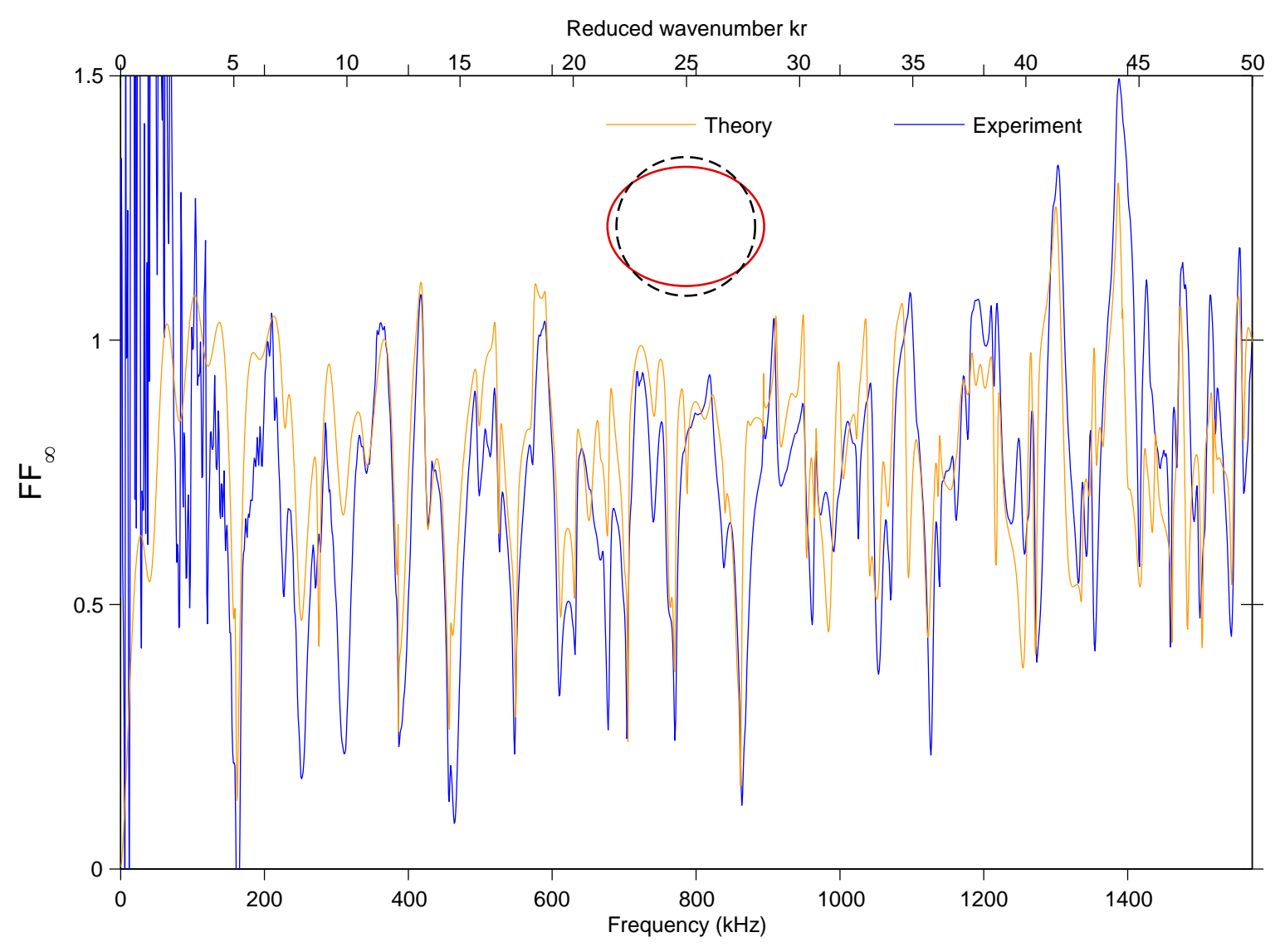

FIG. 9. Comparison between theoretical and experimental form functions for an elliptical cylinder of ratio $b / a=0.7616$ in oblique incidence $\left(\alpha=45^{\circ}\right)$ for an extended frequency range.

\section{CONCLUSION AND PERSPECTIVES}

The scattering of a plane acoustic wave by infinite elastic cylinder of elliptical cross section has been studied from a modal formalism, including symmetry considerations of the scatterer. The use of group representation theory leads to an infinite system of algebraic equations for each irreducible representation $A_{1}, A_{2}, B_{1}, B_{2}$, where the unknown scattering coefficients are uncoupled. A classification of resonances of the infinite elastic elliptical cylinder has been provided: they lie in four distinct families associated with the four irreducible representations of the symmetry group $\mathcal{C}_{2 v}$ of the infinite elliptical cylinder. The splitting up of resonances is emphasized and algebraic considerations permit to understand this phenomenon. This method significantly simplifies the numerical treatment of the problem and provides a physical interpretation in terms of symmetry. The computations can be carried 
out at high frequency and also in case of large eccentricities when rapid variations of the curvature radius occur.

Furthermore, a series of experiments based on ultrasonic spectroscopy has been performed in the case of aluminium cylinders of elliptical cross section for various eccentricities immersed in water. The experimental form functions are compared with the theoretical ones. The sharp minima corresponding to resonances as well as the variation of the form function due to the interference phenomenon are observed experimentally in a quite good agreement with the theory. Angular diagrams have been also investigated; they confirm experimentally the identification of the mode studied.

The same approach can be also applied to three-dimensional problems such as acoustic scattering by a spheroid, or the study of inner resonant modes of a spheroid in elastodynamics. In both cases, it would be interesting to observe the splitting of resonances in the transition from a sphere to a spheroid due to the symmetry breaking $\mathcal{O}(3) \rightarrow \mathcal{D}_{\infty h}$, theoretically and experimentally. For this purpose, an experimental part will be added to the theoretical studies by ultrasonic spectroscopy for the scattering problem and laser vibrometry for the elastodynamics problem.

\section{Appendix A: System of equations for each irreducible representation}

\section{Representation $A_{1}$}

We express the the boundary conditions in the irreducible representation $A_{1}$. From the rules expressed in section III A, one deduces that $\phi, p_{s}$ and $p_{i n c}$ have to belong to $A_{1}$ while $\psi$ has to belong to $A_{2}$. Using Eqs. (16a) and (16b), one obtains

$$
\begin{gathered}
\phi^{\mathrm{A}_{1}}=\frac{1}{\rho_{s} \omega^{2}} \sum_{n \text { even }} \gamma_{n} A_{n}^{L} J_{n}\left(k_{L} \rho\right) \cos (n \theta), \quad \psi^{\mathrm{A}_{2}}=\frac{i}{\rho_{s} \omega^{2}} \sum_{n \text { even }} \gamma_{n} A_{n}^{T} J_{n}\left(k_{T} \rho\right) \sin (n \theta) \\
p_{s}^{\mathrm{A}_{1}}=\sum_{n \text { even }} i^{n} \gamma_{n} A_{n}^{S} H_{n}^{(1)}(k \rho) \cos (n \theta), \quad p_{\text {inc }}^{\mathrm{A}_{1}}=\sum_{n \text { even }} i^{n} \gamma_{n} J_{n}(k \rho) \cos (n \alpha) \cos (n \theta)
\end{gathered}
$$


where $\gamma_{n}$ is the Neumann factor defined by $\gamma_{0}=1$ and $\gamma_{n}=2$ for $n>0$. Then, the boundary conditions expressed by Eqs. (17) and (19) read

$$
\begin{aligned}
& \sum_{n \text { even }} \gamma_{n}\left[A_{n}^{L}\left(-a_{n}^{L} \cos (n \theta) n_{\rho}+b_{n}^{L} \sin (n \theta) n_{\theta}\right)+i A_{n}^{T}\left(-a_{n}^{T} \cos (n \theta) n_{\rho}+b_{n}^{T} \sin (n \theta) n_{\theta}\right)\right. \\
& \left.+i^{n} A_{n}^{S}\left(-a_{n}^{S} \cos (n \theta) n_{\rho}+b_{n}^{S} \sin (n \theta) n_{\theta}\right)\right]=\sum_{n \text { even }} \gamma_{n}\left[i^{n} \cos (n \alpha)\left(-a_{n}^{i n c} \cos (n \theta) n_{\rho}+b_{n}^{i n c} \sin (n \theta) n_{\theta}\right)\right]
\end{aligned}
$$

$$
\begin{aligned}
& \sum_{n \text { even }} \gamma_{n}\left[A_{n}^{L}\left(c_{n}^{L} \cos (n \theta) n_{\rho}+d_{n}^{L} \sin (n \theta) n_{\theta}\right)+i A_{n}^{T}\left(c_{n}^{T} \cos (n \theta) n_{\rho}+d_{n}^{T} \sin (n \theta) n_{\theta}\right)\right. \\
& \left.+i^{n} A_{n}^{S}\left(c_{n}^{S} \cos (n \theta) n_{\rho}\right)\right]=\sum_{n \text { even }} \gamma_{n}\left[i^{n} \cos (n \alpha)\left(c_{n}^{i n c} \cos (n \theta) n_{\rho}\right)\right] \\
& \sum_{n \text { even }} \gamma_{n}\left[A_{n}^{L}\left(e_{n}^{L} \sin (n \theta) n_{\rho}-f_{n}^{L} \cos (n \theta) n_{\theta}\right)+i A_{n}^{T}\left(e_{n}^{T} \sin (n \theta) n_{\rho}-f_{n}^{T} \cos (n \theta) n_{\theta}\right)\right. \\
& \left.+i^{n} A_{n}^{S}\left(e_{n}^{S} \cos (n \theta) n_{\theta}\right)\right]=\sum_{n \text { even }} \gamma_{n}\left[i^{n} \cos (n \alpha)\left(e_{n}^{i n c} \cos (n \theta) n_{\theta}\right)\right]
\end{aligned}
$$

where the structural functions $a_{n}^{j}, b_{n}^{j}, c_{n}^{j}, d_{n}^{j}, f_{n}^{j}, e_{n}^{j} j \in\{L, T, S, i n c\}$ are given in Appendix B. To overcome the angular dependence, the functions $F_{n}(\theta)$ in parenthesis appearing in Eqs. (A3) are expanded in Fourier series by setting

$$
F_{n}(\theta)=\sum_{p=-\infty}^{+\infty} f_{n, p} e^{i p \theta}, \quad \text { with } f_{n, p}=\frac{1}{2 \pi} \int_{-\pi}^{\pi} F_{n}(\theta) e^{-i p \theta} d \theta
$$

In Eq. (A4), the restriction to the fundamental domain and the parity of $F_{n}(\theta)$ permits one to reduce the domain of integration from $[-\pi, \pi]$ to $[0, \pi / 2]$ and the sum over $p$ from $[-\infty,+\infty]$ to $[0,+\infty]$ with $p$ even. Then Eqs. (A3) lead to the final equations 


$$
\begin{aligned}
& \sum_{n, p \text { even }} \gamma_{n} \gamma_{p}\left[A_{n}^{L} \alpha_{n, p}^{L}+i A_{n}^{T} \alpha_{n, p}^{T}+i^{n} A_{n}^{S} \alpha_{n, p}^{S}\right] \cos (p \theta)=\sum_{n, p \text { even }} \gamma_{n} \gamma_{p}\left[i^{n} \cos (n \alpha) \alpha_{n, p}^{i n c}\right] \cos (p \theta) \\
& \sum_{n, p \text { even }} \gamma_{n} \gamma_{p}\left[A_{n}^{L} \beta_{n, p}^{L}+i A_{n}^{T} \beta_{n, p}^{T}+i^{n} A_{n}^{S} \beta_{n, p}^{S}\right] \cos (p \theta)=\sum_{n, p \text { even }} \gamma_{n} \gamma_{p}\left[i^{n} \cos (n \alpha) \beta_{n, p}^{i n c}\right] \cos (p \theta) \\
& \sum_{n, p \text { even }} \gamma_{n} \gamma_{p}\left[A_{n}^{L} \xi_{n, p}^{L}+i A_{n}^{T} \xi_{n, p}^{T}+i^{n} A_{n}^{S} \xi_{n, p}^{S}\right] \sin (p \theta)=\sum_{n, p \text { even }} \gamma_{n} \gamma_{p}\left[i^{n} \cos (n \alpha) \xi_{n, p}^{\text {inc }}\right] \sin (p \theta)
\end{aligned}
$$

where the Fourier coefficients $\alpha_{n, p}^{(j)}, \beta_{n, p}^{(j)}, \xi_{n, p}^{(j)}, j \in\{L, T, S, i n c\}$ are given in Appendix C.

Proceeding by a similar way, we obtain mutatis mutandis the final equations for the representations $A_{2}, B_{1}$ and $B_{2}$.

\section{Representation $A_{2}$}

We express the boundary conditions in the irreducible representation $A_{2}$ using

$$
\begin{array}{rr}
\phi^{\mathrm{A}_{2}}=\frac{i}{\rho_{s} \omega^{2}} \sum_{n \text { even }} \gamma_{n} A_{n}^{L} J_{n}\left(k_{L} \rho\right) \sin (n \theta), \quad \psi^{\mathrm{A}_{1}}=\frac{1}{\rho_{s} \omega^{2}} \sum_{n \text { even }} \gamma_{n} A_{n}^{T} J_{n}\left(k_{T} \rho\right) \cos (n \theta) \\
p_{s}^{\mathrm{A}_{2}}=i \sum_{n \text { even }} \gamma_{n} i^{n} A_{n}^{S} H_{n}^{(1)}(k \rho) \sin (n \theta), \quad p_{i n c}^{\mathrm{A}_{2}}=\sum_{n \text { even }} \gamma_{n} i^{n} J_{n}(k \rho) \sin (n \alpha) \sin (n \theta)
\end{array}
$$


given by Eqs. (16a) and (16b). They read

$$
\begin{aligned}
& \sum_{n \text { even }} \gamma_{n}\left[A_{n}^{L}\left(a_{n}^{L} \sin (n \theta) n_{\rho}+b_{n}^{L} \cos (n \theta) n_{\theta}\right)+i A_{n}^{T}\left(a_{n}^{T} \sin (n \theta) n_{\rho}+b_{n}^{T} \cos (n \theta) n_{\theta}\right)\right. \\
& \left.+i^{n} A_{n}^{S}\left(a_{n}^{S} \sin (n \theta) n_{\rho}+b_{n}^{S} \cos (n \theta) n_{\theta}\right)\right]=-\sum_{n \text { even }} i \gamma_{n}\left[i^{n} \sin (n \alpha)\left(a_{n}^{i n c} \sin (n \theta) n_{\rho}+b_{n}^{i n c} \cos (n \theta) n_{\theta}\right)\right]
\end{aligned}
$$

$$
\begin{aligned}
& \sum_{n \text { even }} \gamma_{n}\left[A_{n}^{L}\left(c_{n}^{L} \sin (n \theta) n_{\rho}-d_{n}^{L} \cos (n \theta) n_{\theta}\right)+i A_{n}^{T}\left(c_{n}^{T} \sin (n \theta) n_{\rho}-d_{n}^{T} \cos (n \theta) n_{\theta}\right)\right. \\
& \left.+i^{n} A_{n}^{S}\left(c_{n}^{S} \sin (n \theta) n_{\rho}\right)\right]=-\sum_{n \text { even }} i \gamma_{n}\left[i^{n} \sin (n \alpha)\left(c_{n}^{i n c} \sin (n \theta) n_{\rho}\right)\right] \\
& \sum_{n \text { even }} \gamma_{n}\left[A_{n}^{L}\left(e_{n}^{L} \cos (n \theta) n_{\rho}+f_{n}^{L} \sin (n \theta) n_{\theta}\right)+i A_{n}^{T}\left(e_{n}^{T} \cos (n \theta) n_{\rho}+f_{n}^{T} \sin (n \theta) n_{\theta}\right)\right. \\
& \left.-i^{n} A_{n}^{S}\left(e_{n}^{S} \sin (n \theta) n_{\theta}\right)\right]=\sum_{n \text { even }} i \gamma_{n}\left[i^{n} \sin (n \alpha)\left(e_{n}^{i n c} \sin (n \theta) n_{\theta}\right)\right]
\end{aligned}
$$

where the structural functions $a_{n}^{j}, b_{n}^{j}, c_{n}^{j}, d_{n}^{j}, f_{n}^{j}, e_{n}^{j} j \in\{L, T, S, i n c\}$ are given in Appendix B. Then, Eqs. (A8) lead to the final equations

$$
\begin{aligned}
& \sum_{n, p \text { even }} \gamma_{n} \gamma_{p}\left[A_{n}^{L} \kappa_{n, p}^{L}+i A_{n}^{T} \kappa_{n, p}^{T}+i^{n} A_{n}^{S} \kappa_{n, p}^{S}\right] \sin (p \theta)=-\sum_{n, p \text { even }} i \gamma_{n} \gamma_{p}\left[i^{n} \sin (n \alpha) \kappa_{n, p}^{i n c}\right] \sin (p \theta) \\
& \sum_{n, p \text { even }} \gamma_{n} \gamma_{p}\left[A_{n}^{L} \eta_{n, p}^{L}+i A_{n}^{T} \eta_{n, p}^{T}+i^{n} A_{n}^{S} \eta_{n, p}^{S}\right] \sin (p \theta)=-\sum_{n, p \text { even }} i \gamma_{n} \gamma_{p}\left[i^{n} \sin (n \alpha) \eta_{n, p}^{i n c}\right] \sin (p \theta) \\
& \sum_{n, p \text { even }} \gamma_{n} \gamma_{p}\left[A_{n}^{L} \zeta_{n, p}^{L}+i A_{n}^{T} \zeta_{n, p}^{T}-i^{n} A_{n}^{S} \zeta_{n, p}^{S}\right] \cos (p \theta)=\sum_{n, p \text { even }} i \gamma_{n} \gamma_{p}\left[i^{n} \sin (n \alpha) \zeta_{n, p}^{i n c}\right] \cos (p \theta)
\end{aligned}
$$

where the Fourier coefficients $\kappa_{n, p}^{(j)}, \eta_{n, p}^{(j)}, \zeta_{n, p}^{(j)}, j \in\{L, T, S, i n c\}$ are given in Appendix C.

\section{Representation $B_{1}$}

We express the boundary conditions in the irreducible representation $B_{1}$ using

$$
\phi^{\mathrm{B}_{1}}=\frac{2}{\rho_{s} \omega^{2}} \sum_{n \text { odd }} A_{n}^{L} J_{n}\left(k_{L} \rho\right) \cos (n \theta), \quad \psi^{\mathrm{B}_{2}}=\frac{2 i}{\rho_{s} \omega^{2}} \sum_{n \text { odd }} A_{n}^{T} J_{n}\left(k_{T} \rho\right) \sin (n \theta)
$$




$$
p_{s}^{\mathrm{B}_{1}}=2 \sum_{n \text { odd }} i^{n} A_{n}^{S} H_{n}^{(1)}(k \rho) \cos (n \theta), \quad p_{\text {inc }}^{\mathrm{B}_{1}}=2 \sum_{n \text { odd }} i^{n} J_{n}(k \rho) \cos (n \alpha) \cos (n \theta)
$$

given by Eqs. (16c) and (16d). They read

$$
\begin{aligned}
& \sum_{n \text { odd }}\left[A_{n}^{L}\left(-a_{n}^{L} \cos (n \theta) n_{\rho}+b_{n}^{L} \sin (n \theta) n_{\theta}\right)+i A_{n}^{T}\left(-a_{n}^{T} \cos (n \theta) n_{\rho}+b_{n}^{T} \sin (n \theta) n_{\theta}\right)\right. \\
& \left.+i^{n} A_{n}^{S}\left(-a_{n}^{S} \cos (n \theta) n_{\rho}+b_{n}^{S} \sin (n \theta) n_{\theta}\right)\right]=\sum_{n \text { odd }}\left[i^{n} \cos (n \alpha)\left(-a_{n}^{i n c} \cos (n \theta) n_{\rho}+b_{n}^{i n c} \sin (n \theta) n_{\theta}\right)\right]
\end{aligned}
$$

$$
\begin{aligned}
& \sum_{n \text { odd }}\left[A_{n}^{L}\left(c_{n}^{L} \cos (n \theta) n_{\rho}+d_{n}^{L} \sin (n \theta) n_{\theta}\right)+i A_{n}^{T}\left(c_{n}^{T} \cos (n \theta) n_{\rho}+d_{n}^{T} \sin (n \theta) n_{\theta}\right)\right. \\
& \left.+i^{n} A_{n}^{S}\left(c_{n}^{S} \cos (n \theta) n_{\rho}\right)\right]=\sum_{n \text { odd }}\left[i^{n} \cos (n \alpha)\left(c_{n}^{i n c} \cos (n \theta) n_{\rho}\right)\right] \\
& \sum_{n \text { odd }}\left[A_{n}^{L}\left(e_{n}^{L} \sin (n \theta) n_{\rho}-f_{n}^{L} \cos (n \theta) n_{\theta}\right)+i A_{n}^{T}\left(e_{n}^{T} \sin (n \theta) n_{\rho}-f_{n}^{T} \cos (n \theta) n_{\theta}\right)\right. \\
& \left.+i^{n} A_{n}^{S}\left(e_{n}^{S} \cos (n \theta) n_{\theta}\right)\right]=\sum_{n \text { odd }}\left[i^{n} \cos (n \alpha)\left(e_{n}^{i n c} \cos (n \theta) n_{\theta}\right)\right]
\end{aligned}
$$

where the structural functions $a_{n}^{j}, b_{n}^{j}, c_{n}^{j}, d_{n}^{j}, f_{n}^{j}, e_{n}^{j} j \in\{L, T, S, i n c\}$ are given in Appendix B. Then, Eqs. (A12) lead to the final equations

$$
\begin{aligned}
& \sum_{n, p \text { odd }}\left[A_{n}^{L} \alpha_{n, p}^{L}+i A_{n}^{T} \alpha_{n, p}^{T}+i^{n} A_{n}^{S} \alpha_{n, p}^{S}\right] \cos (p \theta)=\sum_{n, p \text { odd }}\left[i^{n} \cos (n \alpha) \alpha_{n, p}^{i n c}\right] \cos (p \theta) \\
& \sum_{n, p \text { odd }}\left[A_{n}^{L} \beta_{n, p}^{L}+i A_{n}^{T} \beta_{n, p}^{T}+i^{n} A_{n}^{S} \beta_{n, p}^{S}\right] \cos (p \theta)=\sum_{n, p \text { odd }}\left[i^{n} \cos (n \alpha) \beta_{n, p}^{i n c}\right] \cos (p \theta) \\
& \sum_{n, p \text { odd }}\left[A_{n}^{L} \xi_{n, p}^{L}+i A_{n}^{T} \xi_{n, p}^{T}+i^{n} A_{n}^{S} \xi_{n, p}^{S}\right] \sin (p \theta)=\sum_{n, p \text { odd }}\left[i^{n} \cos (n \alpha) \xi_{n, p}^{i n c}\right] \sin (p \theta)
\end{aligned}
$$

where the Fourier coefficients $\alpha_{n, p}^{(j)}, \beta_{n, p}^{(j)}, \xi_{n, p}^{(j)}, j \in\{L, T, S, i n c\}$ are given in Appendix C.

\section{Representation $B_{2}$}

We express the boundary conditions in the irreducible representation $B_{2}$ using

$$
\phi^{\mathrm{B}_{2}}=\frac{2 i}{\rho_{s} \omega^{2}} \sum_{n \text { odd }} A_{n}^{L} J_{n}\left(k_{L} \rho\right) \sin (n \theta), \quad \psi^{\mathrm{B}_{1}}=\frac{2}{\rho_{s} \omega^{2}} \sum_{n \text { odd }} A_{n}^{T} J_{n}\left(k_{T} \rho\right) \cos (n \theta)
$$




$$
p_{s}^{\mathrm{B}_{2}}=2 i \sum_{n \text { odd }} i^{n} A_{n}^{S} H_{n}^{(1)}(k \rho) \sin (n \theta), \quad p_{i n c}^{\mathrm{B}_{2}}=2 \sum_{n \text { odd }} i^{n} J_{n}(k \rho) \sin (n \alpha) \sin (n \theta)
$$

given by Eqs. (16c) and (16d). They read

$$
\begin{aligned}
& \sum_{n \text { odd }}\left[A_{n}^{L}\left(a_{n}^{L} \sin (n \theta) n_{\rho}+b_{n}^{L} \cos (n \theta) n_{\theta}\right)+i A_{n}^{T}\left(a_{n}^{T} \sin (n \theta) n_{\rho}+b_{n}^{T} \cos (n \theta) n_{\theta}\right)\right. \\
& \left.+i^{n} A_{n}^{S}\left(a_{n}^{S} \sin (n \theta) n_{\rho}+b_{n}^{S} \cos (n \theta) n_{\theta}\right)\right]=-\sum_{n \text { odd }} i\left[i^{n} \sin (n \alpha)\left(a_{n}^{i n c} \sin (n \theta) n_{\rho}+b_{n}^{i n c} \cos (n \theta) n_{\theta}\right)\right]
\end{aligned}
$$

$$
\begin{aligned}
& \sum_{n \text { odd }}\left[A_{n}^{L}\left(c_{n}^{L} \sin (n \theta) n_{\rho}-d_{n}^{L} \cos (n \theta) n_{\theta}\right)+i A_{n}^{T}\left(c_{n}^{T} \sin (n \theta) n_{\rho}-d_{n}^{T} \cos (n \theta) n_{\theta}\right)\right. \\
& \left.+i^{n} A_{n}^{S}\left(c_{n}^{S} \sin (n \theta) n_{\rho}\right)\right]=-\sum_{n \text { odd }} i\left[i^{n} \sin (n \alpha)\left(c_{n}^{i n c} \sin (n \theta) n_{\rho}\right)\right] \\
& \sum_{n \text { odd }}\left[A_{n}^{L}\left(e_{n}^{L} \cos (n \theta) n_{\rho}+f_{n}^{L} \sin (n \theta) n_{\theta}\right)+i A_{n}^{T}\left(e_{n}^{T} \cos (n \theta) n_{\rho}+f_{n}^{T} \sin (n \theta) n_{\theta}\right)\right. \\
& \left.-i^{n} A_{n}^{S}\left(e_{n}^{S} \sin (n \theta) n_{\theta}\right)\right]=\sum_{n \text { odd }} i\left[i^{n} \sin (n \alpha)\left(e_{n}^{i n c} \sin (n \theta) n_{\theta}\right)\right]
\end{aligned}
$$

where the structural functions $a_{n}^{j}, b_{n}^{j}, c_{n}^{j}, d_{n}^{j}, f_{n}^{j}, e_{n}^{j} j \in\{L, T, S, i n c\}$ are given in Appendix B. Then, Eqs. (A16) lead to the final equations

$$
\begin{aligned}
& \sum_{n, p \text { odd }}\left[A_{n}^{L} \kappa_{n, p}^{L}+i A_{n}^{T} \kappa_{n, p}^{T}+i^{n} A_{n}^{S} \kappa_{n, p}^{S}\right] \sin (p \theta)=-\sum_{n, p \text { odd }} i\left[i^{n} \sin (n \alpha) \kappa_{n, p}^{i n c}\right] \sin (p \theta) \\
& \sum_{n, p \text { odd }}\left[A_{n}^{L} \eta_{n, p}^{L}+i A_{n}^{T} \eta_{n, p}^{T}+i^{n} A_{n}^{S} \eta_{n, p}^{S}\right] \sin (p \theta)=-\sum_{n, p \text { odd }} i\left[i^{n} \sin (n \alpha) \eta_{n, p}^{i n c}\right] \sin (p \theta) \\
& \sum_{n, p \text { odd }}\left[A_{n}^{L} \zeta_{n, p}^{L}+i A_{n}^{T} \zeta_{n, p}^{T}-i^{n} A_{n}^{S} \zeta_{n, p}^{S}\right] \cos (p \theta)=\sum_{n, p \text { odd }} i\left[i^{n} \sin (n \alpha) \zeta_{n, p}^{i n c}\right] \cos (p \theta)
\end{aligned}
$$

where the Fourier coefficients $\kappa_{n, p}^{(j)}, \eta_{n, p}^{(j)}, \zeta_{n, p}^{(j)}, j \in\{L, T, S, i n c\}$ are given in Appendix C.

\section{Appendix B: Structural coefficients}

The structural functions involving Bessel functions are angular dependent. They are given by 


$$
\begin{aligned}
& a_{q}^{S}=\frac{\rho_{s}}{\rho_{f}} x H_{q}^{(1)^{\prime}}(x), \quad b_{q}^{S}=\frac{\rho_{s}}{\rho_{f}} q H_{q}^{(1)}(x), \quad c_{q}^{S}=x_{T}^{2} H_{q}^{(1)}(x), \quad e_{q}^{S}=c_{q}^{S} \\
& a_{q}^{L}=x_{L} J_{q}^{\prime}\left(x_{L}\right), \quad b_{q}^{L}=q J_{q}\left(x_{L}\right), \quad c_{q}^{L}=\left(x_{T}^{2}-2 q^{2}\right) J_{q}\left(x_{L}\right)+2 x_{L} J_{q}^{\prime}\left(x_{L}\right), \\
& d_{q}^{L}=2 q\left(x_{L} J_{q}^{\prime}\left(x_{L}\right)-J_{q}\left(x_{L}\right)\right), \quad f_{q}^{L}=\left(2 x_{L}^{2}-x_{T}^{2}-2 q^{2}\right) J_{q}\left(x_{L}\right)+2 x_{L} J_{q}^{\prime}\left(x_{L}\right), \quad e_{q}^{L}=d_{q}^{L} \\
& a_{q}^{T}=-q J_{q}\left(x_{T}\right), \quad b_{q}^{T}=-x_{T} J_{q}^{\prime}\left(x_{T}\right), \quad c_{q}^{T}=2 q\left(x_{T} J_{q}^{\prime}\left(x_{T}\right)-J_{q}\left(x_{T}\right)\right) \\
& d_{q}^{T}=\left(x_{T}^{2}-2 q^{2}\right) J_{q}\left(x_{T}\right)+2 x_{T} J_{q}^{\prime}\left(x_{T}\right), \quad e_{q}^{T}=d_{q}^{T}, \quad c_{q}^{T}=f_{q}^{T} \\
& a_{q}^{i n c}=-\frac{\rho_{s}}{\rho_{f}} x J_{q}^{\prime}(x), \quad b_{q}^{i n c}=-\frac{\rho_{s}}{\rho_{f}} q J_{q}(x), \quad c_{q}^{i n c}=-x_{T}^{2} J_{q}(x), \quad e_{q}^{i n c}=c_{q}^{i n c}
\end{aligned}
$$

where $x=k r(\theta), x_{j}=k_{j} r(\theta), j \in\{L, T\}, q \in \mathbb{N}$ (even for $A_{1}$ and $A_{2}$ or odd for $B_{1}$ and $B_{2}$ ). 


\section{Appendix C: Fourier coefficients}

The Fourier coefficients are evaluated on the restricted fundamental domain, they are numerically calculated from the following expressions

$$
\begin{aligned}
& \alpha_{q, r}^{(j)}=\frac{2}{\pi} \int_{0}^{\pi / 2}\left(-a_{q}^{(j)} \cos (q \theta) n_{\rho}+b_{q}^{(j)} \sin (q \theta) n_{\theta}\right) \cos (r \theta) d \theta \quad j \in\{L, T, S, i n c\} \\
& \beta_{q, r}^{(j)}=\frac{2}{\pi} \int_{0}^{\pi / 2}\left(c_{q}^{(j)} \cos (q \theta) n_{\rho}+d_{q}^{(j)} \sin (q \theta) n_{\theta}\right) \cos (r \theta) d \theta j \in\{L, T\} \\
& \beta_{q, r}^{(j)}=\frac{2}{\pi} \int_{0}^{\pi / 2}\left(c_{q}^{(j)} \cos (q \theta) n_{\rho}\right) \cos (r \theta) d \theta j \in\{S, i n c\} \\
& \xi_{q, r}^{(j)}=\frac{-2 i}{\pi} \int_{0}^{\pi / 2}\left(e_{q}^{(j)} \sin (q \theta) n_{\rho}-f_{q}^{(j)} \cos (q \theta) n_{\theta}\right) \sin (r \theta) d \theta j \in\{L, T\} \\
& \xi_{q, r}^{j}=\frac{-2 i}{\pi} \int_{0}^{\pi / 2}\left(e_{q}^{j} \cos (q \theta) n_{\theta}\right) \sin (r \theta) d \theta j \in\{S, i n c\} \\
& \kappa_{q, r}^{(j)}=\frac{-2 i}{\pi} \int_{0}^{\pi / 2}\left(a_{q}^{(j)} \sin (q \theta) n_{\rho}+b_{q}^{(j)} \cos (q \theta) n_{\theta}\right) \sin (r \theta) d \theta j \in\{L, T, S, i n c\} \\
& \eta_{q, r}^{(j)}=\frac{-2 i}{\pi} \int_{0}^{\pi / 2}\left(c_{q}^{(j)} \sin (q \theta) n_{\rho}-d_{q}^{(j)} \cos (q \theta) n_{\theta}\right) \sin (r \theta) d \theta j \in\{L, T\} \\
& \eta_{q, r}^{(j)}=\frac{-2 i}{\pi} \int_{0}^{\pi / 2}\left(c_{q}^{(j)} \sin (q \theta) n_{\rho}\right) \sin (r \theta) d \theta j \in\{S, i n c\} \\
& \zeta_{q, r}^{(j)}=\frac{2}{\pi} \int_{0}^{\pi / 2}\left(e_{q}^{(j)} \cos (q \theta) n_{\rho}+f_{q}^{(j)} \sin (q \theta) n_{\theta}\right) \cos (r \theta) d \theta j \in\{L, T\} \\
& \zeta_{q, r}^{(j)}=\frac{2}{\pi} \int_{0}^{\pi / 2}\left(e_{q}^{(j)} \sin (q \theta) n_{\theta}\right) \cos (r \theta) d \theta j \in\{S, i n c\} \\
&
\end{aligned}
$$

$(q, r) \in \mathbb{N}^{2}$ (even for $A_{1}$ and $A_{2}$ or odd for $B_{1}$ and $B_{2}$ ).

\section{REFERENCES}

${ }^{1}$ N. D. Veskler, Resonance acoustic spectroscopy (Springer-Verlag, 1993).

${ }^{2}$ J. J. Bowman, T. B. A. Senior, P. L. E. Uslenghi, and J. S. Asvestas, Electromagnetic and acoustic scattering by simple shapes (North-Holland Pub. Co., 1970).

${ }^{3}$ P. A. Chinnery and V. F. Humphrey, J. Acoust. Soc. Am. 103, 1296 (1998).

${ }^{4}$ A. Pereira, A. Tadeu, and J. Antonio, Wave Motion 36, 23 (2002).

${ }^{5}$ F. Léon, F. Chati, and J. M. Conoir, J. Acoust. Soc. Am. 116, 3600 (2004).

${ }^{6}$ F. Léon, F. Chati, and J. Conoir, in Ultrasonics, 42 (2004) pp. 297-300.

${ }^{7}$ R. Barakat, J. Acoust. Soc. Am. 35, 1990 (1963). 
${ }^{8}$ J. E. Burke and V. Twersky, J. Opt. Soc. Am. 54, 732 (1964).

${ }^{9}$ J. E. Burke, J. Acoust. Soc. Am. 36, 2059 (1964).

${ }^{10}$ V. V. Varadan and Y. H. Pao, J. Acoust. Soc. Am. 60, 556 (September 1976).

${ }^{11}$ V. V. Varadan, J. Acoust. Soc. Am. 63, 1014 (Apr. 1978).

${ }^{12} \mathrm{~V}$. V. Varadan, Elastic wave scattering (Acoustic, Electromagnetic and Elastic Waves Scattering-Focused on the T-Matrix Approach) (Pergamon, New York, 1980).

${ }^{13}$ T. A. K. Pillai, V. V. Varadan, and V. K. Varadan, J. Acoust. Soc. Am. 72, 1032 (September 1982).

${ }^{14}$ M. M. Simon and R. P. Raddlinski, J. Acoust. Soc. Am. 71, 273 (1982).

${ }^{15}$ R. P. Raddlinski and M. M. Simon, J. Acoust. Soc. Am. 93, 2443 (1993).

${ }^{16}$ G. C. Goel and D. L. Jain, J. Acoust. Soc. Am. 69, 371 (1981).

${ }^{17}$ N. D. Veksler, B. Dubus, and A. Lavie, Acoust. Phys. 45, 46 (1999).

${ }^{18}$ P. T. DiPerna and T. K. Stanton, J. Acoust. Soc. Am. 96, 3064 (1994).

${ }^{19}$ P. J. Moser and H. Überall, in Proc. IA, Vol. 71 (IEEE, 1983) pp. 171-172.

${ }^{20}$ S. Ancey, A. Folacci, and P. Gabrielli, J. Phys. A: Math. Gen. 34, 2657 (2001).

${ }^{21} \mathrm{~S}$. Ancey, Résonances en géométrie elliptique: développements asymptotiques exponentiellement améliorés et levée de dégénérescence, Ph.D. thesis, Université de Corse - Pascal Paoli (2000).

${ }^{22}$ S. Ancey, E. Bazzali, P. Gabrielli, and M. Mercier, J. Phys. A: Math. Gen. 46, 435204 (2013).

${ }^{23}$ M. Hamermesh, Group Theory and its Application to Physical Problems (New York: Dover, 1989).

${ }^{24}$ Y. Decanini, A. Folacci, P. Gabrielli, and J. L. Rossi, Journal of Sound and Vibration 221, 785 (1999).

${ }^{25}$ P. Gabrielli and M. Mercier-Finidori, Journal of Sound and Vibration 241, 423 (2001).

${ }^{26}$ L. Landau and E. Lifchitz, Physique théorique, tome \%. Théorie de l'élasticité (Librairie du Globe, ed. MIR, 1990).

${ }^{27} r$ is the mean radius defined in such a way that the perimeter of the ellipse remains constant and equal to $2 \pi$.

${ }^{28} \mathrm{We}$ can note that the comparison remains good out of the $-12 \mathrm{~dB}$ transducers bandwidth given in table III. 
${ }^{29} \mathrm{We}$ limit the imaginary part of the complex $k r$-plane to -0.2 . The resonances having a larger absolute value of their imaginary part are too attenuated to be observed on the form function. 